\title{
Flash vacuum pyrolysis of oxo-stabilised phosphonium ylides containing methoxythiophene and methylthiophene groups
}

\author{
R. Alan Aitken ${ }^{*}$ and Alasdair N. Garnett
}

EaStCHEM School of Chemistry, University of St. Andrews, North Haugh, St. Andrews, Fife, UK KY16 9ST. E-mail: raa@st-and.ac.uk

\begin{abstract}
Ten new oxo-stabilised phosphonium ylides containing substituted thiophene groups, as well as two deuterium-labelled analogues, are prepared and fully characterised. Upon flash vacuum pyrolysis at $800{ }^{\circ} \mathrm{C}$, the simpler examples undergo extrusion of $\mathrm{Ph}_{3} \mathrm{PO}$ coupled with domino cyclisation to give thieno[3,2-b]furan and thieno[3,4$b]$ furan products but the corresponding approach to a thieno[2,3-b]furan fails. One ylide designed to give a thieno[3,4- $b]$ furan instead gives phenylbutadiyne and 2phenyl-3-vinylthiophene at $725^{\circ} \mathrm{C}$ and the mechanism of this unusual process is elucidated by deuterium labelling. Attempts to access more complex heterocyclic products from extended methoxythienyl ylides failed. Two ylides bearing a 3-methyl2-thienylacryloyl group gave 5-benzylbenzothiophene, 5- $\alpha$ methylbenzyl)benzothiophene and fluoreno[3,4-b]thiophene upon FVP at $800{ }^{\circ} \mathrm{C}$ and the mechanism was again elucidated by deuterium labelling.
\end{abstract}

Keywords: Flash vacuum pyrolysis; Phosphonium ylide; Thienofuran; Benzothiophene; Fluorenothiophene; Radical cyclisation

\section{$1 \quad$ Introduction}

Flash vacuum pyrolysis (FVP) is now well established as an important method, both for generation of reactive intermediates and preparation of stable products [1]. As a synthetic method it has had a particular impact in the area of heterocyclic chemistry, allowing convenient formation of many otherwise inaccessible products in high yield [2]. For some years we have made a detailed study of oxo-stabilised phosphonium ylides as suitable precursors for synthesis of fused ring heterocycles by FVP in a process involving thermal extrusion of $\mathrm{Ph}_{3} \mathrm{PO}$ to form an alkyne, simultaneous radical generation, and then a sequence of domino cyclisation processes leading to tri-, tetraand pentacyclic fused ring aromatic heterocycles. Recent examples showing the versatility of the method include pyrolysis of nearly 40 compounds of structure 1 to give the corresponding products 2 (Scheme 1) including representatives of 24 different heterocyclic ring systems [3], cascade processes of up to eight steps [4], and introduction of nitrogen, either by starting from pyridine-containing ylides $\mathbf{3}$ which react to afford 4 [5], or by using a nitrogen-based cyclising radical which unexpectedly leads to both five- and six-membered ring heterocycles as illustrated by pyrolysis of ylide 5 to give both the quinoline $\mathbf{6}$ and the furocarbazole natural product Eustifoline D 7 [6-8]. In terms of the mechanism of these processes, this is illustrated for the case of a generalised ylide in Scheme 2 and is supported by the results of isotopic labelling [9]. 

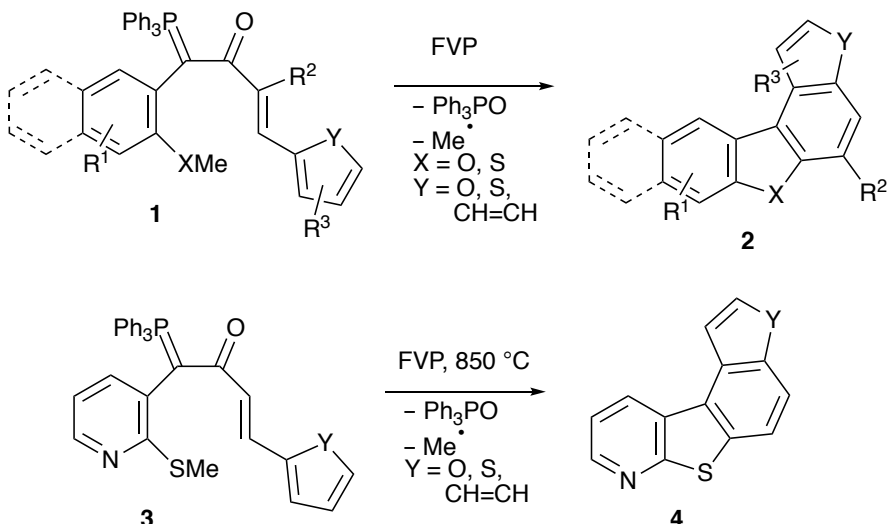<smiles>[Y]1c2ccccc2c2c1ccc1sc3ncccc3c12</smiles>

4

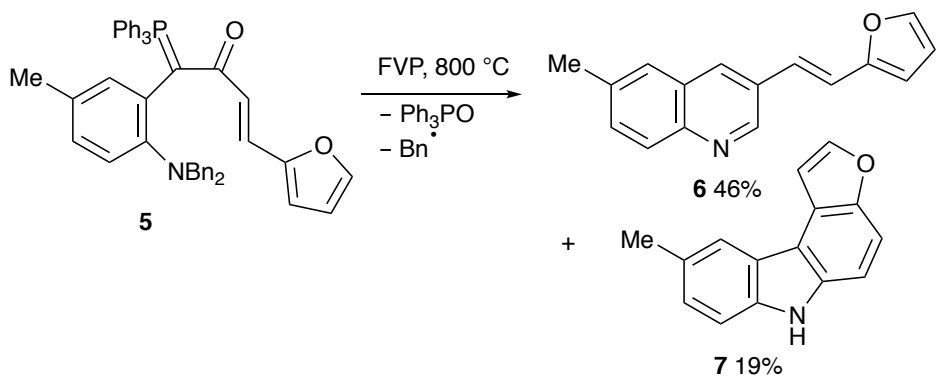

Scheme 1. Previous syntheses of fused ring heterocycles by oxo ylide pyrolysis

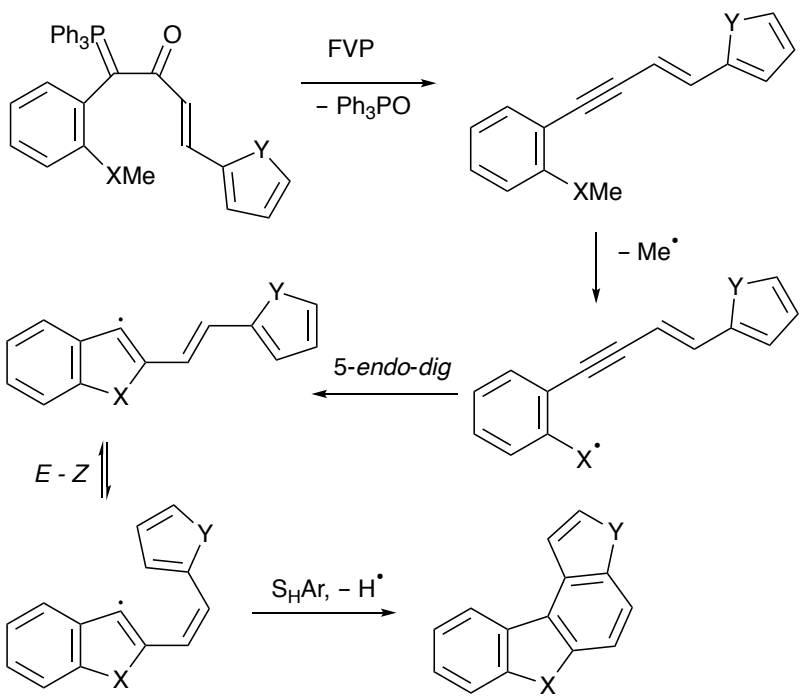

Scheme 2. Typical mechanism for generation of a tetracyclic product

In this paper we report the synthesis and FVP behaviour of a range of thiophene-containing oxo-stabilised ylides. In view of the success in replacing the starting benzene ring of $\mathbf{1}$ with pyridine in $\mathbf{3}$, we were interested to see whether the thermodynamically stable thiophene ring could also be used and thus we examined a range of methoxythienyl ylides as a potential route to fused ring thiophene products. The expected thienofurans are relatively inaccessible heterocycles [10] and typical routes to thieno[3,2-b]furans [11-14] and thieno[3,4-b]furans [14-16] often involve several steps and require certain groups to be present. We have also recently encountered unexpected rearrangements of thiophenes under FVP conditions [17], and we include here also an account of further unexpected thermal processes involving oxo ylides containing a methylthienyl group. 


\subsection{General}

Melting points were recorded on a Reichert hot-stage microscope and are uncorrected. Infra-red spectra were recorded as Nujol mulls for solids and as thin films for liquids on a Perkin Elmer 1710 instrument. NMR spectra were obtained for ${ }^{1} \mathrm{H}$ at 200 or 300 $\mathrm{MHz}$, for ${ }^{13} \mathrm{C}$ at 50 or $75 \mathrm{MHz}$ and for ${ }^{31} \mathrm{P}$ at $121 \mathrm{MHz}$ all using Varian Gemini instruments. ${ }^{2} \mathrm{H}$ NMR spectra were run at $46 \mathrm{MHz}$ in $\mathrm{CHCl}_{3}$. All other spectra were run on solutions in $\mathrm{CDCl}_{3}$ with internal $\mathrm{Me}_{4} \mathrm{Si}$ as reference for ${ }^{1} \mathrm{H}$ and ${ }^{13} \mathrm{C}$ and external $\mathrm{H}_{3} \mathrm{PO}_{4}$ for ${ }^{31} \mathrm{P}$. Chemical shifts are reported in ppm to high frequency of the reference and coupling constants $J$ are in Hz. Mass spectra were obtained on an A. E. I. Kratos MS-50 spectrometer using electron impact at $70 \mathrm{eV}$. GCMS was carried out using a Hewlett Packard 5890A coupled to a Finnigan-Incos 50 mass spectrometer. Elemental analysis was carried out on a Carlo-Erba 1106 elemental analyser.

Flash vacuum pyrolysis (FVP) was carried out as previously reported [18] in a conventional flow system by subliming the starting material through a horizontal quartz tube $(30 \times 2.5 \mathrm{~cm})$ externally heated by a tube furnace to $700-800{ }^{\circ} \mathrm{C}$ and maintained at a pressure of $2-9 \times 10^{-2}$ torr by a rotary vacuum pump. The contact time in the hot zone under these conditions is estimated to be $\sim 10^{-2} \mathrm{~s}$. The apparatus used is illustrated and a detailed experimental procedure is given in a recent publication [19]. Products were collected in a liquid $\mathrm{N}_{2}$ cooled U-shaped trap and purified as noted.

\subsection{Preparation of Simple Methoxythienyl Ylides}

\subsubsection{4-Methoxy-3-thenoyl chloride}

This was prepared by heating a mixture of 4-methoxythiophene-3-carboxylic acid [20] $(3.0 \mathrm{~g}, 19 \mathrm{mmol})$ and thionyl chloride $\left(2.1 \mathrm{~cm}^{3}, 30 \mathrm{mmol}\right)$ under reflux for $2 \mathrm{~h}$ followed by evaporation under reduced pressure and kugelrohr distillation of the residue at 20 Torr to give the product $(2.85 \mathrm{~g}, 85 \%)$ as a pale yellow crystalline solid, mp 47-48 ${ }^{\circ} \mathrm{C}$ (Found: $\mathrm{C}, 40.9 ; \mathrm{H}, 2.6 . \mathrm{C}_{6} \mathrm{H}_{5} \mathrm{ClO}_{2} \mathrm{~S}$ requires $\mathrm{C}, 40.8 ; \mathrm{H}, 2.9 \%$ ); $v_{\max } / \mathrm{cm}^{-1} 1781,1541,1408,1389,1214,1179,1150,1010,911,886,815,789$ and $718 ; \delta_{\mathrm{H}} 8.38(1 \mathrm{H}, \mathrm{d}, J 3,2-\mathrm{H}), 6.33(1 \mathrm{H}, \mathrm{d}, J 3,5-\mathrm{H})$ and $3.90(3 \mathrm{H}, \mathrm{s}, \mathrm{OMe}) ; \delta_{\mathrm{C}} 158.3$ (C), $157.5(\mathrm{C}), 140.5(\mathrm{CH}), 126.5(\mathrm{C}), 99.0(\mathrm{CH})$ and $58.2(\mathrm{Me}) ; \mathrm{m} / z 178\left({ }^{37} \mathrm{Cl}_{-\mathrm{M}}^{+}\right.$, $11 \%), 176\left({ }^{35} \mathrm{Cl}_{-1} \mathrm{M}^{+}, 29\right), 141(100), 126(17), 98(5)$ and $53(14)$.

\subsubsection{4-Trideuteriomethoxy-3-thenoyl chloride}

This was prepared as in 2.2.1 using 4-trideuteriomethoxythiophene-3-carboxylic acid [20] $(0.38 \mathrm{~g}, 2.4 \mathrm{mmol})$ and thionyl chloride $\left(0.73 \mathrm{~cm}^{3}, 10 \mathrm{mmol}\right)$ to give the product $(0.18 \mathrm{~g}, 40 \%)$ as a pale yellow crystalline solid, mp $44-47^{\circ} \mathrm{C} ; \delta_{\mathrm{H}} 8.37(1 \mathrm{H}, \mathrm{d}, J 4,2-$ $\mathrm{H})$ and $6.33(1 \mathrm{H}, \mathrm{d}, J 4,5-\mathrm{H})$.

\subsection{3. [(3-Methoxy-2-thenoyl)benzylidene] triphenylphosphorane $\mathbf{8}$}

A suspension of benzyltriphenylphosphonium chloride $(10 \mathrm{~g}, 26.0 \mathrm{mmol})$ in dry THF $\left(70 \mathrm{~cm}^{3}\right)$ was stirred under $\mathrm{N}_{2}$ while a solution of butyllithium in hexanes $\left(10.4 \mathrm{~cm}^{3}\right.$, $2.5 \mathrm{M}, 26.0 \mathrm{mmol})$ was added. The resulting deep red solution was stirred for a further $2 \mathrm{~h}$ before adding a solution of 3-methoxy-2-thenoyl chloride [21] (2.30 g, $13.0 \mathrm{mmol})$ in dry THF $\left(20 \mathrm{~cm}^{3}\right)$. After stirring for a further $24 \mathrm{~h}$ the mixture was added to water $\left(100 \mathrm{~cm}^{3}\right)$ and extracted with diethyl ether $\left(2 \times 150 \mathrm{~cm}^{3}\right)$ and ethyl acetate $\left(2 \times 150 \mathrm{~cm}^{3}\right)$. The combined extracts were washed with water $\left(100 \mathrm{~cm}^{3}\right)$, dried and evaporated to give a yellow oil which was triturated with diethyl ether to 
give a yellow solid. This was dissolved in ethyl acetate $\left(250 \mathrm{~cm}^{3}\right)$, the hot solution filtered and the solid obtained on evaporation recrystallised to give the product (3.03 $\mathrm{g}, 47 \%$ ) as bright yellow crystals, mp 189-190 ${ }^{\circ} \mathrm{C}$ (Found: C, 75.4; H, 5.0. $\mathrm{C}_{31} \mathrm{H}_{25} \mathrm{O}_{2} \mathrm{PS}$ requires $\left.\mathrm{C}, 75.6 ; \mathrm{H}, 5.1 \%\right) ; v_{\max } / \mathrm{cm}^{-1} 1592,1543,1510,1351,1237$, $1104,998,967,849,750$ and $690 ; \delta_{\mathrm{H}} 7.75-7.63(6 \mathrm{H}, \mathrm{m}, \mathrm{Ph}), 7.60-7.25(9 \mathrm{H}, \mathrm{m}, \mathrm{Ph})$, $7.02(1 \mathrm{H}, \mathrm{d}, J 6,5-\mathrm{H}), 6.92-6.85(5 \mathrm{H}, \mathrm{m}, \mathrm{Ph}), 6.66(1 \mathrm{H}, \mathrm{d}, J 6,4-\mathrm{H})$, and $3.38(3 \mathrm{H}, \mathrm{s}$, $\mathrm{OMe}) ; \delta_{\mathrm{C}} 177.4$ (d, $\left.J 6, \mathrm{CO}\right), 155.0$ (thienyl C-3), 138.1 (d, $J 11, \mathrm{C}-1$ of C-Ph), 134.9 $(\mathrm{d}, J 4,2 \mathrm{CH}), 133.9$ (d, $J 10, \mathrm{C}-2$ of PPh), $131.5(\mathrm{~d}, J$ 2, C-4 of PPh), $128.6(\mathrm{~d}, J 12$, C-3 of PPh), $127.3(\mathrm{~d}, J$ 92, C-1 of PPh), $127.0(2 \mathrm{CH}), 124.9(\mathrm{CH}), 124.7(\mathrm{CH}), 123.3$ $\left(\mathrm{d}, J 14\right.$, thienyl C-2), 116.3 (thienyl C-4), $74.0(\mathrm{~d}, J 109, \mathrm{C}=\mathrm{P})$ and $58.2(\mathrm{OMe}) ; \delta_{\mathrm{P}}$ +15.9; m/z 492 (M+, 30\%), 461 (5), 277 (58), 262 (100), 230 (19), 214 (24), 183 (45), 165 (12), 141 (19), 108 (13) and 91 (6).

\subsection{4 [(3-Methoxy-2-thenoyl)-1-propylidene]triphenylphosphorane 9}

This was prepared as in 2.2.3 using propyltriphenylphosphonium bromide (10 g, 26.0 mmol), butyllithium in hexanes $\left(10.4 \mathrm{~cm}^{3}, 2.5 \mathrm{M}, 26.0 \mathrm{mmol}\right)$ and 3-methoxy-2thenoyl chloride [21] $(2.30 \mathrm{~g}, 13.0 \mathrm{mmol})$ to give the product $(1.71 \mathrm{~g}, 30 \%)$ as redorange crystals, mp $222-224{ }^{\circ} \mathrm{C}$ (Found: $\mathrm{C}, 72.7 ; \mathrm{H}, 5.4 . \mathrm{C}_{27} \mathrm{H}_{25} \mathrm{O}_{2} \mathrm{PS}$ requires $\mathrm{C}$, 73.0; H, 5.7\%); $v_{\max } / \mathrm{cm}^{-1} 1542,1494,1445,1235,1181,1104,1047,1016,930,834$, 744,710 and $695 ; \delta_{\mathrm{H}} 7.85-7.60(6 \mathrm{H}, \mathrm{m}, \mathrm{Ph}), 7.60-7.35(9 \mathrm{H}, \mathrm{m}, \mathrm{Ph}), 7.13(1 \mathrm{H}, \mathrm{d}, J 6$, 5-H), $6.89(1 \mathrm{H}, \mathrm{d}, J 6,4-\mathrm{H}), 3.90(3 \mathrm{H}, \mathrm{s}, \mathrm{OMe}), 2.19\left(2 \mathrm{H}, \mathrm{dq}, J 23,7, \mathrm{CH}_{2}\right)$ and 0.73 $\left(3 \mathrm{H}, \mathrm{t}, J 7, \mathrm{CH}_{3}\right) ; \delta_{\mathrm{C}} 177.5(\mathrm{~d}, J 7, \mathrm{CO}), 153.7$ (thienyl C-3), $133.8(\mathrm{~d}, J 10, \mathrm{C}-2$ of PPh), 131.5 (d, $J$ 2, C-4 of PPh), 128.7 (d, $J$ 12, C-3 of PPh), 127.7 (d, $J$ 91, C-1 of $\mathrm{PPh}$ ), 123.8 (d, $J$ 10, thienyl C-2), $123.7(\mathrm{CH}), 116.8$ (thienyl C-4), 71.2 (d, $J$ 100, $\mathrm{C}=\mathrm{P}), 58.9(\mathrm{OMe}), 20.7\left(\mathrm{~d}, J 13, \mathrm{CH}_{2}\right)$ and $14.2(\mathrm{Me}) ; \delta_{\mathrm{P}}+16.5 ; \mathrm{m} / \mathrm{z} 444\left(\mathrm{M}^{+}, 7 \%\right)$, 429 (34), 287 (8), 277 (22), 262 (100), 201 (7), 183 (35), 152 (7), 141 (23), 127 (7) and $108(12)$.

\subsection{5 [(4-Methoxy-3-thenoyl)benzylidene] triphenylphosphorane $\mathbf{1 0}$}

This was prepared as in 2.2.3 using benzyltriphenylphosphonium chloride (6.14 g, $15.8 \mathrm{mmol})$, butyllithium in hexanes $\left(6.34 \mathrm{~cm}^{3}, 2.5 \mathrm{M}, 15.8 \mathrm{mmol}\right)$ and 4-methoxy-3thenoyl chloride $(1.40 \mathrm{~g}, 7.9 \mathrm{mmol})$ to give the product $(3.35 \mathrm{~g}, 86 \%)$ as very pale yellow crystals, mp $166-168{ }^{\circ} \mathrm{C}$ (Found: $\mathrm{C}, 75.3 ; \mathrm{H}, 5.0 . \mathrm{C}_{31} \mathrm{H}_{25} \mathrm{O}_{2} \mathrm{PS}$ requires $\mathrm{C}$, 75.6; H, 5.1\%); $v_{\max } / \mathrm{cm}^{-1} 1592,1491,1432,1238,1216,1158,1107,1030,1001$, $970,874,854,761,750$ and $690 ; \delta_{\mathrm{H}} 7.71-7.53(6 \mathrm{H}, \mathrm{m}, \mathrm{Ph}), 7.52-7.32(9 \mathrm{H}, \mathrm{m}, \mathrm{Ph})$, $6.98(1 \mathrm{H}, \mathrm{d}, J 3,2-\mathrm{H}), 6.84(5 \mathrm{H}$, br s, $\mathrm{Ph}), 5.97(1 \mathrm{H}, \mathrm{d}, J 3,5-\mathrm{H})$ and $3.61(3 \mathrm{H}, \mathrm{s}$, OMe); $\delta_{\mathrm{C}} 180.6(\mathrm{~d}, J 8, \mathrm{CO}), 156.8$ (thienyl C-4), 138.0 (d, $J 12, \mathrm{C}-1$ of C-Ph), 135.7 (d, $J$ 13, thienyl C-3), $134.5(\mathrm{~d}, J 5,2 \mathrm{CH}), 133.8(\mathrm{~d}, J 10, \mathrm{C}-2$ of PPh), $131.5(\mathrm{~d}, J 2$, C-4 of PPh), 128.6 (d, $J$ 12, C-3 of PPh), 127.0 (d, $J$ 91, C-1 of PPh), $127.0(2 \mathrm{CH})$, $124.9(\mathrm{CH}), 124.5$ (thienyl C-2), 95.7 (thienyl C-5), $74.1(\mathrm{~d}, J 107, \mathrm{C}=\mathrm{P})$ and 57.2 (OMe); $\delta_{\mathrm{P}}+16.0 ; m / z 492\left(\mathrm{M}^{+}, 25 \%\right), 461$ (3), 277 (40), 262 (100), 236 (6), 201 (10), 183 (39), $141(24)$ and $69(45)$

\subsection{6 [(4-Trideuteriomethoxy-3-thenoyl)benzylidene] triphenylphosphorane $\mathbf{1 1}$}

This was prepared as in 2.2.3 using benzyltriphenylphosphonium chloride $(0.78 \mathrm{~g}, 2$ mmol), butyllithium in hexanes $\left(0.8 \mathrm{~cm}^{3}, 2.5 \mathrm{M}, 2 \mathrm{mmol}\right)$ and 4-trideuteriomethoxy3-thenoyl chloride $(0.18 \mathrm{~g}, 1 \mathrm{mmol})$ to give a pale yellow oil which was triturated with diethyl ether to give pale yellow needles which were recrystallised from ethyl acetate to give the product $(0.3 \mathrm{~g}, 61 \%)$ as very pale yellow crystals, mp 163.5-168 
${ }^{\circ} \mathrm{C} ; \delta_{\mathrm{H}} 7.71-7.53(6 \mathrm{H}, \mathrm{m}, \mathrm{Ph}), 7.52-7.32(9 \mathrm{H}, \mathrm{m}, \mathrm{Ph}), 6.98(1 \mathrm{H}, \mathrm{d}, J 3,2-\mathrm{H}), 6.84$ $(5 \mathrm{H}$, br s, $\mathrm{Ph})$ and $5.84(1 \mathrm{H}, \mathrm{d}, J 3,5-\mathrm{H}) ; \delta_{\mathrm{P}}+15.9$.

\subsection{7 [(4-Methoxy-3-thenoyl)-1-propylidene]triphenylphosphorane $\mathbf{1 2}$}

This was prepared as in 2.2.3 using propyltriphenylphosphonium bromide $(6.14 \mathrm{~g}$, $15.9 \mathrm{mmol})$, butyllithium in hexanes $\left(6.35 \mathrm{~cm}^{3}, 2.5 \mathrm{M}, 15.9 \mathrm{mmol}\right)$ and 4-methoxy-3thenoyl chloride $(1.40 \mathrm{~g}, 7.9 \mathrm{mmol})$ to give the product $(3.09 \mathrm{~g}, 88 \%)$ as very pale yellow needles, mp 226-228 ${ }^{\circ} \mathrm{C}$ (Found: $\mathrm{C}, 72.8 ; \mathrm{H}, 5.6 . \mathrm{C}_{27} \mathrm{H}_{25} \mathrm{O}_{2} \mathrm{PS}$ requires $\mathrm{C}$, 73.0; H, 5.7\%); $v_{\max } / \mathrm{cm}^{-1} 1545,1495,1364,1321,1276,1198,1178,1153,1103$, $1031,990,942,882,855,827,776,695$ and $623 ; \delta_{\mathrm{H}} 7.82-7.60(6 \mathrm{H}, \mathrm{m}, \mathrm{Ph}), 7.60$ $7.38(9 \mathrm{H}, \mathrm{m}, \mathrm{Ph}), 7.21(1 \mathrm{H}, \mathrm{d}, J 3,2-\mathrm{H}), 6.18(1 \mathrm{H}, \mathrm{d}, J 3,5-\mathrm{H}), 3.87(3 \mathrm{H}, \mathrm{s}, \mathrm{OMe})$, $1.98\left(2 \mathrm{H}, \mathrm{dq}, J 23,7, \mathrm{CH}_{2}\right)$ and $0.63\left(3 \mathrm{H}, \mathrm{t}, J 7, \mathrm{CH}_{3}\right) ; \delta_{\mathrm{C}} 181.5(\mathrm{~d}, J 8, \mathrm{CO}), 156.6$ (thienyl C-4), 136.3 (d, $J$ 13, thienyl C-3), 133.8 (d, $J 10, \mathrm{C}-2$ of PPh), $131.6(\mathrm{~d}, J 2$, C-4 of PPh), 128.7 (d, $J$ 12, C-3 of PPh), 127.3 (d, $J$ 89, C-1 of PPh), 121.8 (thienyl C-2), 96.1 (thienyl C-5), $70.0(\mathrm{~d}, J 100, \mathrm{C}=\mathrm{P}), 57.6(\mathrm{OMe}), 21.0\left(\mathrm{~d}, J 13, \mathrm{CH}_{2}\right)$ and $18.8(\mathrm{Me}) ; \delta_{\mathrm{P}}+17.3 ; \mathrm{m} / \mathrm{z} 444\left(\mathrm{M}^{+}, 10 \%\right), 429$ (24), 410 (6), $386(8), 368(10), 262$ (67), 236 (24), 183 (23), 137 (21), 123 (16), 111 (18), 97 (32), 81 (52) and 69 (100).

\subsection{8 [(2-Methoxy-3-thenoyl)benzylidene] triphenylphosphorane $\mathbf{1 3}$}

This was prepared as in 2.2.3 using benzyltriphenylphosphonium chloride (10.00 g, $26 \mathrm{mmol})$, butyllithium in hexanes $\left(10.4 \mathrm{~cm}^{3}, 2.5 \mathrm{M}, 26 \mathrm{mmol}\right)$ and 2-methoxy-3thenoyl chloride [22] $(2.30 \mathrm{~g}, 13 \mathrm{mmol})$ in dry THF $\left(10 \mathrm{~cm}^{3}\right)$. After stirring for a further $24 \mathrm{~h}$ the mixture was added to water $\left(200 \mathrm{~cm}^{3}\right)$ and extracted with diethyl ether $\left(2 \times 100 \mathrm{~cm}^{3}\right)$ and ethyl acetate $\left(2 \times 75 \mathrm{~cm}^{3}\right)$. The combined extracts were washed with water $\left(100 \mathrm{~cm}^{3}\right)$, dried, decolourised with charcoal and evaporated to give a pale yellow solid which was recrystallised from ethyl acetate to give the product (1.07 g, 17\%) as pale yellow prisms, mp $182-185^{\circ} \mathrm{C}$ (Found: $\mathrm{C}, 75.3 ; \mathrm{H}, 5.5$. $\mathrm{C}_{31} \mathrm{H}_{25} \mathrm{O}_{2} \mathrm{PS}$ requires $\left.\mathrm{C}, 75.6 ; \mathrm{H}, 5.1 \%\right) ; v_{\max } / \mathrm{cm}^{-1} 1548,1495,1440,1398,1334$, $1267,1224,1105,1002,969,933,762$ and $702 ; \delta_{\mathrm{H}} 7.63-7.57(6 \mathrm{H}, \mathrm{m}, \mathrm{Ph}), 7.50-7.32$ $(9 \mathrm{H}, \mathrm{m}, \mathrm{Ph}), 6.88-6.79(5 \mathrm{H}, \mathrm{m}, \mathrm{Ph}), 6.69(1 \mathrm{H}, \mathrm{d}, J 6,4-\mathrm{H}), 6.25(1 \mathrm{H}, \mathrm{d}, J 6,5-\mathrm{H})$ and $3.52(3 \mathrm{H}, \mathrm{s}, \mathrm{OMe}) ; \delta_{\mathrm{C}} 180.4(\mathrm{~d}, J 6, \mathrm{CO}), 163.5$ (thienyl C-2), $138.2(\mathrm{~d}, J 12, \mathrm{C}-1$ of C-Ph), 134.4 (d, $J 4,2 \mathrm{CH}), 133.8$ (d, $J$ 10, C-2 of PPh), 131.5 (d, $J$ 2, C-4 of PPh), $128.6(\mathrm{~d}, J 12, \mathrm{C}-3$ of PPh), $127.9(\mathrm{CH}), 127.4(\mathrm{~d}, J$ 91, C-1 of PPh), $127.0(2 \mathrm{CH})$, $124.4(\mathrm{CH}), 124.0(\mathrm{~d}, J 12$, thienyl C-3), 109.3 (thienyl C-5), $74.0(\mathrm{~d}, J 109, \mathrm{C}=\mathrm{P})$ and $61.5(\mathrm{OMe}) ; \delta_{\mathrm{P}}+15.6 ; \mathrm{m} / \mathrm{z} 492\left(\mathrm{M}^{+}, 54 \%\right), 461$ (22), 379 (9), 277 (52), 262 (100), 214 (28), 199 (23) and 183 (46).

\subsection{Pyrolysis of Simple Methoxythienyl Ylides}

\subsubsection{FVP of [(3-methoxy-2-thenoyl) benzylidene] triphenylphosphorane $\mathbf{8}$}

FVP of the title compound $(1.10 \mathrm{~g})$ at $800^{\circ} \mathrm{C}$ and $3.0-5.0 \times 10^{-2}$ Torr gave a brown oily solid at the furnace exit. Column chromatography of this using diethyl etherhexane (1:9) as eluant to give a brown oil which solidified with time. This was then distilled at $160{ }^{\circ} \mathrm{C} / 0.01$ Torr to give a clear yellow oil which solidified on cooling. This was recrystallised from ethanol to give the product, 2-phenylthieno[3,2-b]furan $14(0.20 \mathrm{~g}, 45 \%)$ as pale brown plates, $\mathrm{mp} 115-118^{\circ} \mathrm{C}$ (Found: $\mathrm{C}, 72.1 ; \mathrm{H}, 4.1$. $\mathrm{C}_{12} \mathrm{H}_{8} \mathrm{OS}$ requires $\mathrm{C}, 72.0 ; \mathrm{H}, 4.0 \%$ ) (HRMS: found $\mathrm{M}^{+}$, 200.0292. $\mathrm{C}_{12} \mathrm{H}_{8} \mathrm{OS}$ requires M, 200.0296); $v_{\max } / \mathrm{cm}^{-1} 1594,1335,1304,1163,1043,1014,873,827,737$ and 647; $\delta_{\mathrm{H}} 7.76(2 \mathrm{H}, \mathrm{d}, \mathrm{J}$ 7, Ph), $7.43(2 \mathrm{H}, \mathrm{t}, \mathrm{J}$ 7, Ph), $7.32(1 \mathrm{H}, \mathrm{d}, \mathrm{J}$ 7, Ph), 7.20 (1H, d, J 5, 6- 
H), $7.15(1 \mathrm{H}, \mathrm{d}, \mathrm{J} 5,5-\mathrm{H})$ and $7.05(1 \mathrm{H}, \mathrm{s}, 3-\mathrm{H}) ; \delta_{\mathrm{C}} 158.0(\mathrm{C}), 157.8(\mathrm{C}), 131.3(\mathrm{C})$, $129.0(2 \mathrm{CH}), 128.0(\mathrm{CH}), 125.4(\mathrm{CH}), 125.3(\mathrm{C}), 124.1(2 \mathrm{CH}), 111.1(\mathrm{CH})$ and 101.2 $(\mathrm{CH}) ; \mathrm{m} / z 200\left(\mathrm{M}^{+}, 72 \%\right), 186$ (24), 171 (100), 146 (18), 127 (13), 102 (39), 85 (24) and $77(25)$.

\subsubsection{FVP of [(3-methoxy-2-thenoyl)-1-propylidene] triphenylphosphorane 9}

FVP of the title compound $(0.64 \mathrm{~g})$ at $800{ }^{\circ} \mathrm{C}$ and $3.0-5.0 \times 10^{-2}$ Torr gave a brown oily solid at the furnace exit. Column chromatography of this using diethyl etherhexane $(1: 9)$ as eluant to give a pale brown oil, $(0.10 \mathrm{~g})$ which was found by ${ }^{1} \mathrm{H}$ NMR, ${ }^{13} \mathrm{C}$ NMR and GCMS to be a moderately complex mixture of products including triphenylphosphine and benzothiophene indicating some carbon skeleton fragmentation. However, two of the expected products were seen:

1. major product (28\%): 2-vinylthieno[3,2-b]furan 15; $\delta_{\mathrm{H}} 7.13(1 \mathrm{H}, \mathrm{d}, J 5,6-\mathrm{H}), 7.00$ $(1 \mathrm{H}, \mathrm{d}, J 5,5-\mathrm{H}), 6.54(1 \mathrm{H}, \mathrm{s}, 3-\mathrm{H}), 6.52(1 \mathrm{H}, \mathrm{dd}, J 16,11$, vinyl-H), $5.78(1 \mathrm{H}, \mathrm{d}, J$ 16 , vinyl-H) and $5.21\left(1 \mathrm{H}, \mathrm{d}, J 11\right.$, vinyl-H); $\delta_{\mathrm{C}} 157.1(\mathrm{C}), 156.2(\mathrm{C}), 131.4(\mathrm{CH})$, $125.2(\mathrm{CH}), 124.8(\mathrm{C}), 112.3\left(\mathrm{CH}_{2}\right), 110.2(\mathrm{CH})$ and $103.6(\mathrm{CH}) ; \mathrm{m} / \mathrm{z} 150\left(\mathrm{M}^{+}\right.$, 100\%), 121 (43), 96 (47), 78 (17), 75 (10), 70 (20) and 45 (15);

2. minor product $(9 \%)$ : 2-ethylthieno[3,2-b]furan 16; $\delta_{\mathrm{H}} 7.24(1 \mathrm{H}, \mathrm{d}, J 5,6-\mathrm{H}), 7.05$ $(1 \mathrm{H}, \mathrm{d}, J 5,5-\mathrm{H}), 6.32(1 \mathrm{H}, \mathrm{s}, 3-\mathrm{H}), 2.75\left(2 \mathrm{H}, \mathrm{d}, J 8, \mathrm{CH}_{2}\right)$ and $1.27\left(3 \mathrm{H}, \mathrm{t}, J 8, \mathrm{CH}_{3}\right)$; $\mathrm{m} / \mathrm{z} 152\left(\mathrm{M}^{+}, 40 \%\right), 137$ (100), 109 (10), 97 (9), 69 (13), 65 (12), 53 (11), 4518$)$ and 39 (19).

\subsubsection{FVP of [(4-methoxy-3-thenoyl)-1-propylidene]triphenylphosphorane $\mathbf{1 2}$}

FVP of the title compound $(0.5 \mathrm{~g})$ at $800{ }^{\circ} \mathrm{C}$ and $5.0-9.0 \times 10^{-2}$ Torr gave an oily brown solid at the furnace exit. Column chromatography of this using diethyl etherhexane (1:9) as eluant gave a yellow oil which was kugelrohr distilled to give the product, 2-vinylthieno[3,4-b]furan $17(0.04 \mathrm{~g}, 25 \%)$ as an unstable pale yellow oil, bp (oven temp.) $170{ }^{\circ} \mathrm{C}$ at 0.7 Torr (HRMS: Found $\mathrm{M}^{+}, 150.0133 . \mathrm{C}_{8} \mathrm{H}_{6} \mathrm{OS}$ requires $\mathrm{M}$, 150.0139); $\delta_{\mathrm{H}} 6.87(1 \mathrm{H}, \mathrm{d}, J 2,4-\mathrm{H}), 6.68(1 \mathrm{H}, \mathrm{d}, J 2,6-\mathrm{H}), 6.52(1 \mathrm{H}, \mathrm{dd}, J 18,10$, vinyl-H), $6.24(1 \mathrm{H}, \mathrm{s}, 3-\mathrm{H}), 5.88(1 \mathrm{H}, \mathrm{d}, J 18$, vinyl-H) and $5.38(1 \mathrm{H}, \mathrm{d}, J 10$, vinyl$\mathrm{H}) ; \delta_{\mathrm{C}} 163.1(\mathrm{C}), 156.5(\mathrm{C}), 136.0(\mathrm{C}), 125.9(\mathrm{CH}), 116.2(\mathrm{CH}), 107.6\left(\mathrm{CH}_{2}\right), 101.4$ $(\mathrm{CH})$ and $94.6(\mathrm{CH}) ; \mathrm{m} / z 150\left(\mathrm{M}^{+}, 23 \%\right), 121$ (18), 105 (12), 85 (19), 71 (21), 57 (56) and 43 (100).

\subsubsection{FVP of [(4-methoxy-3-thenoyl)benzylidene] triphenylphosphorane $\mathbf{1 0}$}

FVP of the title compound $(0.5 \mathrm{~g})$ at $725^{\circ} \mathrm{C}$ and $5.0-9.0 \times 10^{-2}$ Torr gave an oily brown solid at the furnace exit. Column chromatography of this using diethyl etherhexane (1:19) as eluant gave two fractions;

1. A mixture of two products, partially separable by preparative TLC;

(a) 2-phenyl-3-vinylthiophene 18 (HRMS: Found $\mathrm{M}^{+}, 186.0498 . \mathrm{C}_{12} \mathrm{H}_{10} \mathrm{~S}$ requires $\mathrm{M}$, 186.0503); $\delta_{\mathrm{H}} 7.62(2 \mathrm{H}, \mathrm{d}, J 7, \mathrm{ArH}), 7.45$ (1H, s, ArH), 7.4-7.25 (3H, m, ArH), 7.13 $(1 \mathrm{H}, \mathrm{s}, \mathrm{ArH}), 6.69(1 \mathrm{H}, \mathrm{dd}, J 18,11$, vinyl-H), $5.62(1 \mathrm{H}, \mathrm{d}, J 18$, vinyl-H) and 5.23 $\left(1 \mathrm{H}, \mathrm{d}, J 11\right.$, vinyl-H); $\delta_{\mathrm{C}} 145.1(\mathrm{C}), 141.5(\mathrm{C}), 134.6(\mathrm{C}), 131.3(\mathrm{CH}), 129.1(2 \mathrm{CH})$, $127.9(\mathrm{CH}), 126.1(2 \mathrm{CH}), 122.1(\mathrm{CH}), 120.8(\mathrm{CH})$ and $113.9\left(\mathrm{CH}_{2}\right) ; \mathrm{m} / \mathrm{z} 186\left(\mathrm{M}^{+}\right.$, 100\%), 171 (8), 152 (9), 141 (6), 115 (13) and 102 (1);

(b) 1-phenyl-1,3-butadiyne 19 (HRMS: Found $\mathrm{M}^{+}$, 126.0474. $\mathrm{C}_{10} \mathrm{H}_{6}$ requires $\mathrm{M}$, 126.0470); $v_{\max } / \mathrm{cm}^{-1} 2200(\mathrm{C} \equiv \mathrm{C}) ; \delta_{\mathrm{H}} 7.52(2 \mathrm{H}, \mathrm{d}, \mathrm{ArH}), 7.4-7.3(3 \mathrm{H}, \mathrm{m}, \mathrm{ArH})$ and $2.45(1 \mathrm{H}, \mathrm{s}, \mathrm{CH})$ [Lit. [23] 7.42-7.2 (5H), $2.37(1 \mathrm{H})$ ]; $\delta_{\mathrm{C}} 133.0(2 \mathrm{CH}), 129.7(\mathrm{CH})$, $128.7(2 \mathrm{CH}), 121.3(\mathrm{C}), 75.5(\mathrm{C} \equiv \mathrm{C}), 73.6(\mathrm{C} \equiv \mathrm{C}), 71.4(\mathrm{C} \equiv \mathrm{CH})$ and $68.3(\mathrm{C} \equiv \mathrm{C})[$ Lit. 
[23] 132.7, 129.5, 128.4, 120.9, 75.3, 73.5, 71.3, 68.1]; m/z $126\left(\mathrm{M}^{+}, 78 \%\right), 98$ (16), 87 (13), 74 (57) and 50 (100);

2. 1-(4-methoxy-3-thienyl)-2-phenylethyne 20 (HRMS: Found $\mathrm{M}^{+}, 214.0457$. $\mathrm{C}_{13} \mathrm{H}_{10} \mathrm{OS}$ requires $\left.\mathrm{M}, 214.0452\right) ; \delta_{\mathrm{H}} 7.60-7.52(2 \mathrm{H}, \mathrm{m}, \mathrm{Ph}), 7.42(1 \mathrm{H}, \mathrm{d}, J 4,2-\mathrm{H})$, 7.38-7.30 (3H, m, Ph), $6.22(1 \mathrm{H}, \mathrm{d}, J 4,5-\mathrm{H})$ and $3.88(3 \mathrm{H}, \mathrm{s}, \mathrm{OMe}) ; \delta_{\mathrm{C}} 158.2(\mathrm{CO})$, $131.9(2 \mathrm{CH}), 128.4(4 \mathrm{CH}), 123.4(\mathrm{C}), 115.0(\mathrm{C}), 96.6(\mathrm{CH}), 91.5(\mathrm{C} \equiv \mathrm{C}), 82.1(\mathrm{C} \equiv \mathrm{C})$ and $58.0(\mathrm{OMe}) ; \mathrm{m} / \mathrm{z} 214\left(\mathrm{M}^{+}, 100 \%\right), 184(10), 171$ (25), 141 (50), $126(50), 115$ (43), 98 (18) and $86(21)$.

\subsubsection{FVP of [(4-trideuteriomethoxy-3-thenoyl)benzylidene] triphenylphosphorane 11}

FVP of the title compound $(0.3 \mathrm{~g})$ at $725^{\circ} \mathrm{C}$ and $3.0-7.0 \times 10^{-2}$ Torr gave a yellowbrown oily solid at the furnace exit. Column chromatography of this using diethyl ether-hexane (1:9) as eluant gave mainly 1-(4-trideuteriomethoxy-3-thienyl)-2phenylethyne $21(0.1 \mathrm{~g}, 76 \%)$ as a brown oil, $\delta_{\mathrm{H}} 7.42(1 \mathrm{H}, \mathrm{d}, J 4,2-\mathrm{H})$ and $6.22(1 \mathrm{H}$, $\mathrm{d}, J 4,5-\mathrm{H}) ; \delta_{\mathrm{D}} 3.88\left(\mathrm{CD}_{3} \mathrm{O}\right) ; \mathrm{m} / z 217\left(\mathrm{M}^{+}, 100 \%\right), 171$ (19), 144 (34), 140 (8), 126 (22) and 117 (5), together with small amounts of 1-phenylbutadiyne 19 and 4deuterio-3-(2,2-dideuteriovinyl)-2-phenylthiophene 22.

\subsubsection{Repyrolysis of product $\mathbf{2 1}$ at $800{ }^{\circ} \mathrm{C}$}

FVP of the above product $(0.1 \mathrm{~g})$ at $800^{\circ} \mathrm{C}$ and $3.0-7.0 \times 10^{-2}$ Torr gave a yellowbrown oily solid at the furnace exit. ${ }^{1} \mathrm{H}$ NMR, ${ }^{2} \mathrm{H}$ NMR and GCMS analysis indicated the presence of 1-phenyl-1,3-butadiyne $19, \delta_{\mathrm{H}} 2.45(\mathrm{C} \equiv \mathrm{CH}) ; m / z 126\left(\mathrm{M}^{+}, 100 \%\right), 98$ (16), 87 (12), 76 (21), 74 (30), 63 (22) and 50 (22), and 4-deuterio-3-(2,2dideuteriovinyl)-2-phenylthiophene $22 ; \delta_{\mathrm{H}} 6.67(1 \mathrm{H}, \mathrm{br} \mathrm{s}$, vinyl- $\mathrm{H}) ; \delta_{\mathrm{D}} 7.50(1 \mathrm{D}, \mathrm{s}$, ArD), 5.68 (1D, s, vinyl-D) and 5.34 (1D, s, vinyl-D); m/z $189\left(\mathrm{M}^{+}, 100 \%\right), 172$ (9), 154 (15), 144 (12), 121 (15), 117 (23), 103 (23) and 77 (30).

\subsection{Preparation of Methoxythienyl Cinnamoyl Ylides}

\subsubsection{3-Methoxy-2-thiophenemethanol 23 [24]}

A solution of methyl 3-methoxythiophene-2-carboxylate [25] (2.0 g, $12 \mathrm{mmol})$ in dry diethyl ether $\left(75 \mathrm{~cm}^{3}\right)$ was added dropwise to a stirred suspension of lithium aluminium hydride $(0.33 \mathrm{~g}, 9 \mathrm{mmol})$ in dry diethyl ether $\left(75 \mathrm{~cm}^{3}\right)$ under $\mathrm{N}_{2}$. The reaction mixture was heated under reflux for $4 \mathrm{~h}$ then cooled. Water $\left(50 \mathrm{~cm}^{3}\right)$ was added cautiously with stirring. The mixture was extracted with diethyl ether $(3 \times 50$ $\mathrm{cm}^{3}$ ), the combined extracts washed with water, dried with anhydrous $\mathrm{Na}_{2} \mathrm{CO}_{3}$, and evaporated to give the product $(1.38 \mathrm{~g}, 80 \%)$ as a clear, colourless oil, unstable to distillation. (HRMS: Found $\mathrm{M}^{+} 144.0240 . \mathrm{C}_{6} \mathrm{H}_{8} \mathrm{O}_{2} \mathrm{~S}$ requires $M, 144.0245$ ); $v_{\max } / \mathrm{cm}^{-1}$ $3392,1559,1382,1252,1154,1116,1000,872,707$ and $667 ; \delta_{\mathrm{H}} 7.14(1 \mathrm{H}, \mathrm{d}, J 6,5-$ $\mathrm{H}), 6.83(1 \mathrm{H}, \mathrm{d}, J 6,4-\mathrm{H}), 4.70\left(2 \mathrm{H}, \mathrm{s}, \mathrm{CH}_{2}\right), 3.85(3 \mathrm{H}, \mathrm{s}, \mathrm{OMe})$ and $2.15(1 \mathrm{H}, \mathrm{s}, \mathrm{OH})$; $\delta_{\mathrm{C}} 155.1(\mathrm{C}-3), 123.3(\mathrm{C}-5), 119.5(\mathrm{C}-2), 116.4(\mathrm{C}-4), 58.9\left(\mathrm{CH}_{2}\right)$ and $56.1(\mathrm{OMe})$; m/z $144\left(\mathrm{M}^{+}, 79 \%\right), 127$ (100), 115 (7), 111 (28), 100 (6), 97 (8), 85 (11) and 75 (33).

\section{4 .2 (3-Methoxy-2-thienylmethyl)triphenylphosphonium chloride $\mathbf{2 4}$}

A solution of 3-methoxy-2-thiophenemethanol $23(13.45 \mathrm{~g}, 93 \mathrm{mmol})$ and triphenylphosphine $(60.0 \mathrm{~g}, 228 \mathrm{mmol})$ in dry acetonitrile $\left(60 \mathrm{~cm}^{3}\right)$ was stirred under $\mathrm{N}_{2}$ at $70^{\circ} \mathrm{C}$ while dry carbon tetrachloride $\left(9.0 \mathrm{~cm}^{3}, 93 \mathrm{mmol}\right)$ was added steadily via a syringe. A vigorous exothermic reaction resulted. The solution was heated under 
reflux for a further $2 \mathrm{~h}$, during which time a creamy white precipitate formed. This was filtered off, washed with cold acetonitrile and recrystallised from acetonitrile to give the product $(27.60 \mathrm{~g}, 70 \%)$ as colourless prisms, mp $233-234.5^{\circ} \mathrm{C}$; (Found: $\mathrm{C}$, 67.9; H, 5.0. $\mathrm{C}_{24} \mathrm{H}_{22} \mathrm{ClOPS}$ requires $\left.\mathrm{C}, 67.8 ; \mathrm{H}, 5.2 \%\right) ; v_{\max } / \mathrm{cm}^{-1} 1587,1554,1439$, $1389,1260,1112,1049,997,928,812,738$ and $691 ; \delta_{\mathrm{H}} 7.98-7.84(3 \mathrm{H}, \mathrm{m}, \mathrm{Ph}), 7.82-$ $7.64(12 \mathrm{H}, \mathrm{m}, \mathrm{Ph}), 7.33(1 \mathrm{H}, \mathrm{dd}, J 6,3,5-\mathrm{H}), 6.91(1 \mathrm{H}, \mathrm{d}, J 6,4-\mathrm{H}), 4.98(2 \mathrm{H}, \mathrm{d}, J 11$, $\mathrm{CH}_{2} \mathrm{P}$ ) and $3.48(3 \mathrm{H}, \mathrm{s}, \mathrm{OMe}) ; \delta_{\mathrm{C}} 159.3(\mathrm{~d}, J 9, \mathrm{C}-3), 136.6(\mathrm{~d}, J 2, \mathrm{C}-4$ of $\mathrm{Ph}), 135.4$ $(\mathrm{d}, J 10, \mathrm{C}-2$ of Ph), 131.5 (d, $J 13, \mathrm{C}-3$ of Ph), 127.0 (d, $J$ 5, C-5), 119.6 (d, $J 85, \mathrm{C}-1$ of $\mathrm{Ph}), 116.9$ (d, $J 3, \mathrm{C}-4), 103.8$ (d, $J 10, \mathrm{C}-2), 58.9(\mathrm{OMe})$ and 22.9 (d, $J 53, \mathrm{CH}_{2}$ ); $\delta_{\mathrm{P}}+20.6$.

\subsubsection{4-Methoxy-3-thiophenemethanol 27 [24]}

This was prepared as in 2.4.1 using methyl 4-methoxythiophene-3-carboxylate [20] $(0.40 \mathrm{~g}, 2.3 \mathrm{mmol})$ and lithium aluminium hydride $(0.10 \mathrm{~g}, 2.6 \mathrm{mmol})$ to give the product $(0.3 \mathrm{~g}, 91 \%)$ as a clear, colourless oil, bp (oven temp.) $70{ }^{\circ} \mathrm{C}$ at 0.6 Torr (HRMS: Found $\mathrm{M}^{+} 144.0239 . \mathrm{C}_{6} \mathrm{H}_{8} \mathrm{O}_{2} \mathrm{~S}$ requires $\left.M, 144.0245\right) ; v_{\max } / \mathrm{cm}^{-1} 3382,2933$, $1562,1479,1413,1348,1208,1179,1133,1040,857,781,731$ and $602 ; \delta_{\mathrm{H}} 7.20(1 \mathrm{H}$, $\mathrm{d}, J 3,2-\mathrm{H}), 6.43(1 \mathrm{H}, \mathrm{d}, J 3,5-\mathrm{H}), 4.57\left(2 \mathrm{H}, \mathrm{s}, \mathrm{CH}_{2}\right), 3.83(3 \mathrm{H}, \mathrm{s}, \mathrm{OMe})$ and 2.19 $(1 \mathrm{H}, \mathrm{s}, \mathrm{OH}) ; \delta_{\mathrm{C}} 156.3(\mathrm{C}-4), 132.8(\mathrm{C}-3), 122.1(\mathrm{C}-2), 97.1(\mathrm{C}-5), 58.3$ and 57.4; $\mathrm{m} / \mathrm{z}$ $144\left(\mathrm{M}^{+}, 100 \%\right), 127$ (32), 115 (34), 111 (35), 101 (10), 97 (10), 84 (9), 73 (9) and 55 (7).

\section{4 .4 (4-Methoxy-3-thienylmethyl)triphenylphosphonium chloride $\mathbf{2 8}$}

This was prepared as in 2.4.2 using 4-methoxy-3-thiophenemethanol 27 (3.5 g, 24 mmol), triphenylphosphine $(12.59 \mathrm{~g}, 48 \mathrm{mmol})$, acetonitrile $\left(30 \mathrm{~cm}^{3}\right)$ and carbon tetrachloride $\left(2.34 \mathrm{~cm}^{3}, 24 \mathrm{mmol}\right)$ to give the product $(5.3 \mathrm{~g}, 52 \%)$ as colourless prisms, mp 266-267 ${ }^{\circ} \mathrm{C}$ (dec.); (Found: $\mathrm{C}, 67.5 ; \mathrm{H}, 5.1 . \mathrm{C}_{24} \mathrm{H}_{22} \mathrm{ClOPS}$ requires $\mathrm{C}$, 67.8; H, 5.2\%); $v_{\max } / \mathrm{cm}^{-1} 2022,1588,1547,1439,1345,1257,1227,1200,1172$, 1046, 997, 891, 866, 824, 767, 751, 721 and 694; $\delta_{\mathrm{H}} 7.88-7.50(15 \mathrm{H}, \mathrm{m}, \mathrm{Ph}), 7.38$ $(1 \mathrm{H}, \mathrm{m}, \mathrm{H}-2), 6.02(1 \mathrm{H}, \mathrm{d}, J 5, \mathrm{H}-5), 5.27\left(2 \mathrm{H}, \mathrm{d}, J 19, \mathrm{CH}_{2} \mathrm{P}\right)$ and $3.24(3 \mathrm{H}, \mathrm{s}, \mathrm{OMe})$; $\delta_{\mathrm{C}} 156.2(\mathrm{~d}, J 2, \mathrm{C}-4), 135.2(\mathrm{~d}, J$ 2, C-4 of Ph), 134.5 (d, $J 10, \mathrm{C}-2$ of Ph), 130.3 (d, $J$ 12, C-3 of Ph), 127.4 (d, J 8, C-2), 118.2 (d, J 87, C-1 of Ph), 117.5 (d, J 9, C-3), 96.9 $(\mathrm{C}-5), 57.2(\mathrm{OMe})$ and $22.8\left(\mathrm{~d}, J 50, \mathrm{CH}_{2} \mathrm{P}\right) ; \delta_{\mathrm{P}}+21.2$.

\subsection{5 [(3-Methoxy-2-thienyl)(3-phenylpropenoyl)methylene] triphenylphosphorane 25}

A suspension of (3-methoxy-2-thienylmethyl)triphenylphosphonium chloride 24 (5.0 $\mathrm{g}, 11.8 \mathrm{mmol})$ in dry THF $\left(50 \mathrm{~cm}^{3}\right)$ was stirred under $\mathrm{N}_{2}$ while a solution of butyllithium in hexanes $\left(4.7 \mathrm{~cm}^{3}, 2.5 \mathrm{M}, 11.8 \mathrm{mmol}\right)$ was added. The resulting deep red solution was stirred for a further $2 \mathrm{~h}$ before adding a solution of 3phenylpropenoyl chloride $(0.98 \mathrm{~g}, 5.9 \mathrm{mmol})$ in dry THF $\left(10 \mathrm{~cm}^{3}\right)$. After stirring for a further $12 \mathrm{~h}$ the mixture was added to water $\left(100 \mathrm{~cm}^{3}\right)$ and extracted with diethyl ether $\left(2 \times 100 \mathrm{~cm}^{3}\right)$ and ethyl acetate $\left(2 \times 200 \mathrm{~cm}^{3}\right)$. The combined extracts were washed with water $\left(100 \mathrm{~cm}^{3}\right)$, dried and evaporated to give a red oil which crystallised on cooling with dry ice to give a red-brown crystalline solid. This was recrystallised from ethyl acetate to give the product $(1.97 \mathrm{~g}, 64 \%)$ as yellow-brown prisms, mp 206-209 ${ }^{\circ} \mathrm{C}$ (dec.) (Found: $\mathrm{C}, 76.1 ; \mathrm{H}$, 5.2. $\mathrm{C}_{33} \mathrm{H}_{27} \mathrm{O}_{2} \mathrm{PS}$ requires $\mathrm{C}$, 76.4; $\mathrm{H}, 5.3 \%) ; v_{\max } / \mathrm{cm}^{-1} 1631,1559,1520,1437,1382,1348,1266,1202,1110,1065$, $974,888,828,738$ and 689; $\delta_{\mathrm{H}} 7.69-7.59(6 \mathrm{H}, \mathrm{m}, \mathrm{Ph}), 7.52-7.34(12 \mathrm{H}, \mathrm{m}, \mathrm{ArH}, \mathrm{Ph}$ 
and vinyl-H), 7.29-7.18 (3H, m, ArH), $7.07(1 \mathrm{H}, \mathrm{d}, J 16$, vinyl-H), $6.99(1 \mathrm{H}, \mathrm{dd}, J 6$, 1, 5'-H), $6.50\left(1 \mathrm{H}, \mathrm{d}, J 6,4^{\prime}-\mathrm{H}\right)$ and $3.38(3 \mathrm{H}, \mathrm{s}, \mathrm{OMe}) ; \delta_{\mathrm{C}} 182.0(\mathrm{~d}, J 9, \mathrm{CO}), 155.8$ (d, $J$ 6, thienyl C-3), $137.2(\mathrm{C}-1$ of C-Ph), $134.8(\mathrm{CH}), 133.8(\mathrm{~d}, J 10, \mathrm{C}-2 \mathrm{of} \mathrm{PPh})$, $131.6(\mathrm{~d}, J 2, \mathrm{C}-4$ of PPh), $128.5(2 \mathrm{CH}), 128.4(\mathrm{~d}, J 13, \mathrm{C}-3$ of PPh), $128.1(2 \mathrm{CH})$, $127.8(\mathrm{CH}), 126.9(\mathrm{~d}, J$ 90, C-1 of PPh), $126.2(\mathrm{~d}, J 12,-\mathrm{CH}=), 124.0(\mathrm{~d}, J 3$, thienyl C-5), $117.8(\mathrm{~d}, J 13$, thienyl C-2), $115.9(\mathrm{~d}, J 2$, thienyl C-4), $62.3(\mathrm{~d}, J 113, \mathrm{C}=\mathrm{P})$ and $58.1(\mathrm{OMe}) ; \delta_{\mathrm{P}}+17.8 ; \mathrm{m} / \mathrm{z} 518\left(\mathrm{M}^{+}, 19 \%\right), 503$ (3), 487 (6), 368 (5), 277 (100), 262 (39), 240 (17), 201 (20), 183 (38) and 152 (12).

\subsection{6 [(3-Methoxy-2-thienyl)(3-(5-methyl-2-thienyl)propenoyl)methylene] tri- -phenylphosphorane 26}

This was prepared as in 2.4.5 using (3-methoxy-2thienylmethyl)triphenylphosphonium chloride $24(9.0 \mathrm{~g}, 21 \mathrm{mmol})$, butyllithium in hexanes $\left(8.4 \mathrm{~cm}^{3}, 2.5 \mathrm{M}, 21 \mathrm{mmol}\right)$ and 3-(5-methyl-2-thienyl)propenoyl chloride [26] (2.05 g, $10.5 \mathrm{mmol})$ to give the product $(0.97 \mathrm{~g}, 23 \%)$ as yellow-orange needles, mp 184-185.5 ${ }^{\circ} \mathrm{C}$ (Found: $\mathrm{C}, 71.1 ; \mathrm{H}, 5.0 . \mathrm{C}_{32} \mathrm{H}_{27} \mathrm{O}_{2} \mathrm{PS}_{2}$ requires $\mathrm{C}, 71.4 ; \mathrm{H}, 5.1 \%$ ); $v_{\max } / \mathrm{cm}^{-1} 1620,1551,1502,1361,1209,1107,1067,962,883,803,747,720$ and $692 ; \delta_{\mathrm{H}} 7.75-7.67(6 \mathrm{H}, \mathrm{m}, \mathrm{Ph}), 7.67-7.34(10 \mathrm{H}, \mathrm{m}, \mathrm{Ph}$ and vinyl-H), $7.02(1 \mathrm{H}, \mathrm{dd}, J$ 6, 2, 5'-H), $6.83(1 \mathrm{H}, \mathrm{d}, J 5,3-\mathrm{H}), 6.79(1 \mathrm{H}, \mathrm{d}, J 16$, vinyl-H), $6.60(1 \mathrm{H}, \mathrm{d}, J 5,4-\mathrm{H})$, $6.52\left(1 \mathrm{H}, \mathrm{d}, J 6,4^{\prime}-\mathrm{H}\right), 3.40(3 \mathrm{H}, \mathrm{s}, \mathrm{OMe})$ and $2.42(3 \mathrm{H}, \mathrm{s}, \mathrm{ArMe}) ; \delta_{\mathrm{C}} 182.0(\mathrm{~d}, J 8$, CO), 155.8 (d, J 5, MeO-thienyl C-3), 140.7 (C), 140.5 (C), 133.8 (d, J 10, C-2 of $\mathrm{PPh}), 131.6$ (d, $J$ 2, C-4 of PPh), $128.6(\mathrm{CH}), 128.5(\mathrm{~d}, J$ 13, C-3 of PPh), $128.3(\mathrm{CH})$, $127.0(\mathrm{~d}, J$ 91, C-1 of PPh), $126.0(\mathrm{CH}), 124.4(\mathrm{~d}, J 11,-\mathrm{C}=), 124.1(\mathrm{~d}, J 4, \mathrm{MeO}-$ thienyl C-5), 117.8 (d, $J$ 12, MeO-thienyl C-2), 115.9 (d, $J$ 2, MeO-thienyl C-4), 61.9 $(\mathrm{d}, J 115, \mathrm{C}=\mathrm{P}), 58.1(\mathrm{OMe})$ and $15.7(\mathrm{Me}) ; \delta_{\mathrm{P}}+17.8 ; \mathrm{m} / z 538\left(\mathrm{M}^{+}, 18 \%\right), 507,(6)$, 277 (18), 262 (40), 183 (28), 151 (28), 111 (33), 91 (72), 71 (65) and 57 (100).

\subsection{7 [(4-Methoxy-3-thienyl)(3-phenylpropenoyl)methylene] triphenylphosphorane}

\section{9}

This was prepared as in 2.4.5 using (4-methoxy-3thienylmethyl)triphenylphosphonium chloride $28(5.0 \mathrm{~g}, 11.8 \mathrm{mmol})$, butyllithium in hexanes $\left(4.7 \mathrm{~cm}^{3}, 2.5 \mathrm{M}, 11.8 \mathrm{mmol}\right)$ and 3-phenylpropenoyl chloride $(0.98 \mathrm{~g}, 5.9$ $\mathrm{mmol})$ to give a yellow crystalline powder which was recrystallised from ethyl acetate to give the product $(2.24 \mathrm{~g}, 73 \%)$ as yellow prisms, mp $238-240{ }^{\circ} \mathrm{C}$ (dec.) (Found: C, 76.1; H, 5.2. $\mathrm{C}_{33} \mathrm{H}_{27} \mathrm{O}_{2} \mathrm{PS}$ requires C, 76.4; H, 5.3\%); $v_{\max } / \mathrm{cm}^{-1} 1627,1545,1511$, 1437, 1348, 1186, 1152, 1107, 1071, 1032, 976, 934, 858, 848, 783, 762, 751, 726 and $694 ; \delta_{\mathrm{H}} 7.69-7.58(6 \mathrm{H}, \mathrm{m}, \mathrm{Ph}), 7.51-7.38(12 \mathrm{H}, \mathrm{m}, \mathrm{ArH}, \mathrm{Ph}$ and vinyl-H), 7.28$7.17(3 \mathrm{H}, \mathrm{m}, \mathrm{ArH}), 6.97\left(1 \mathrm{H}, \mathrm{dd}, J 4,3,2^{\prime}-\mathrm{H}\right), 6.93(1 \mathrm{H}, \mathrm{d}, J 16$, vinyl-H), $5.84(1 \mathrm{H}$, $\left.\mathrm{d}, J 4,5^{\prime}-\mathrm{H}\right)$ and $3.31(3 \mathrm{H}, \mathrm{s}, \mathrm{OMe}) ; \delta_{\mathrm{C}} 180.7$ (d, $J$ 8, CO), 157.9 (thienyl C-4), 137.3 (C-1 of C-Ph), $134.5(\mathrm{CH}), 133.9$ (d, $J$ 10, C-2 of PPh), 131.6 (d, $J 2$, C-4 of PPh), $129.4(\mathrm{~d}, J 11$, thienyl C-3), $128.5(2 \mathrm{CH}), 128.4(\mathrm{~d}, J 12, \mathrm{C}-3$ of PPh), $128.1(2 \mathrm{CH})$, $127.8(\mathrm{CH}), 127.1(\mathrm{~d}, J$ 8, thienyl C-2), $126.9(\mathrm{~d}, J$ 91, C-1 of PPh), $126.2(\mathrm{~d}, J 12$, $\mathrm{CH}=), 95.3$ (thienyl C-5), $66.1(\mathrm{~d}, J 111, \mathrm{C}=\mathrm{P})$ and $56.7(\mathrm{OMe}) ; \delta_{\mathrm{P}}+15.5 ; m / z 518$ $\left(\mathrm{M}^{+}, 21 \%\right), 386(9), 368(20), 326$ (6), 277 (7), 262 (38), 236 (35), 208 (11), 183 (20), 152 (16), 131 (20), $111(30), 97$ (55), 83 (67), 69 (97) and 57 (100).

\subsection{Preparation of Methylthienylacryloyl Ylides}

\subsubsection{3-(3-Methyl-2-thienyl)propenoyl chloride $\mathbf{3 3}$}


A mixture of 3-(3-methyl-2-thienyl)propenoic acid [27] (5.00 g, $30 \mathrm{mmol})$ and oxalyl chloride $\left(3.92 \mathrm{~cm}^{3}, 45 \mathrm{mmol}\right)$ was heated under reflux for $2 \mathrm{~h}$. The oxalyl chloride was removed under reduced pressure and the residue kugelrohr distilled to give the product $\left(5.47 \mathrm{~g}, 98 \%\right.$ ) as lemon-yellow needles, mp $64-66^{\circ} \mathrm{C}$ (Found: $\mathrm{C}, 51.8 ; \mathrm{H}, 3.5$. $\mathrm{C}_{8} \mathrm{H}_{7} \mathrm{ClOS}$ requires C, 51.5; H, 3.8\%); $v_{\max } / \mathrm{cm}^{-1} 1731,1592,1412,1274,1246,1088$, $1045,965,936,800,742,696,655$ and $629 ; \delta_{\mathrm{H}} 7.97(1 \mathrm{H}, \mathrm{d}, J 16$, vinyl-H), $7.42(1 \mathrm{H}$, $\mathrm{d}, J 7,5-\mathrm{H}), 6.94(1 \mathrm{H}, \mathrm{d}, J 7,4-\mathrm{H}), 6.34(1 \mathrm{H}, \mathrm{d}, J 16$, vinyl-H) and $2.39(3 \mathrm{H}, \mathrm{s}, \mathrm{Me})$; $\delta_{\mathrm{C}} 165.9(\mathrm{CO}), 145.4(\mathrm{C}), 141.3(\mathrm{CH}), 132.8(\mathrm{C}), 131.9(\mathrm{CH}), 130.5(\mathrm{CH}), 119.5$

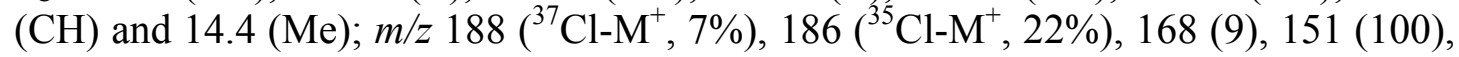
123 (25), 97 (5) and 79 (11).

\subsection{2 [(3-(3-Methyl-2-thienyl)propenoyl)(2-}

methylthiophenyl)methylene] triphenylphosphorane 35

A suspension of (2-methylthiobenzyl)triphenylphosphonium bromide 31 (8.0 g, 16.7 mmol) in dry THF $\left(70 \mathrm{~cm}^{3}\right)$ was stirred under $\mathrm{N}_{2}$ while a solution of butyllithium in hexanes $\left(6.7 \mathrm{~cm}^{3}, 2.5 \mathrm{M}, 16.7 \mathrm{mmol}\right)$ was added. The resulting deep red solution was stirred for a further $2 \mathrm{~h}$ before adding a solution of 3-(3-methyl-2-thienyl)propenoyl chloride $33(1.56 \mathrm{~g}, 8.3 \mathrm{mmol})$ in dry THF $\left(10 \mathrm{~cm}^{3}\right)$. After stirring for a further $18 \mathrm{~h}$ the mixture was added to water $\left(200 \mathrm{~cm}^{3}\right)$ and extracted with diethyl ether $(2 \times 100$ $\left.\mathrm{cm}^{3}\right)$ and ethyl acetate $\left(2 \times 100 \mathrm{~cm}^{3}\right)$. The combined extracts were washed with water $\left(100 \mathrm{~cm}^{3}\right)$, dried and evaporated to give an orange solid which was recrystallised from ethyl acetate to give the product $(3.90 \mathrm{~g}, 86 \%)$ as bright yellow-orange prisms, $\mathrm{mp}$ 220-222 ${ }^{\circ} \mathrm{C}$ (Found: $\mathrm{C}, 74.4 ; \mathrm{H}, 5.1 . \mathrm{C}_{34} \mathrm{H}_{29} \mathrm{OPS}_{2}$ requires $\mathrm{C}, 74.4 ; \mathrm{H}, 5.3 \%$ ); $v_{\max } / \mathrm{cm}^{-}$ ${ }^{1} 1614,1500,1435,1355,1197,1099,1078,978,961,921,852,790,755,714$ and $699 ; \delta_{\mathrm{H}} 7.82-7.52(6 \mathrm{H}, \mathrm{m}, \mathrm{Ph}), 7.63(1 \mathrm{H}, \mathrm{d}, J 16$, vinyl-H), 7.52-7.26 (9H, m, Ph), $7.08\left(1 \mathrm{H}, \mathrm{t}, J 8,4{ }^{\prime}-\mathrm{H}\right), 7.00\left(1 \mathrm{H}, \mathrm{d}, J 8,6{ }^{\prime}-\mathrm{H}\right), 6.95(1 \mathrm{H}, \mathrm{d}, J 7,5-\mathrm{H}), 6.90(1 \mathrm{H}, \mathrm{d}, J 8$, 3'-H), $6.80\left(1 \mathrm{H}, \mathrm{t}, J 8,5^{\prime}-\mathrm{H}\right), 6.72(1 \mathrm{H}, \mathrm{d}, J 7,4-\mathrm{H}), 6.44(1 \mathrm{H}, \mathrm{d}, J 16$, vinyl-H) and $2.21\left(6 \mathrm{H}, \mathrm{s}, \mathrm{Me}\right.$ and SMe); $\delta_{\mathrm{C}} 180.3(\mathrm{~d}, J 6, \mathrm{CO}), 145.2\left(\mathrm{~d}, J 5, \mathrm{MeSC}_{6} \mathrm{H}_{4} \mathrm{C}-2\right), 137.9$ (C), $136.5(\mathrm{CH}), 136.3(\mathrm{~d}, J 3, \mathrm{CH}), 135.0$ (d, $\left.J 11, \mathrm{MeSC}_{6} \mathrm{H}_{4} \mathrm{C}-1\right), 134.0(\mathrm{~d}, J 10, \mathrm{C}-$ 2 of PPh), 131.6 (d, J 3, C-4 of PPh), 130.9 (C), 128.4 (d, $J$ 12, C-3 of PPh), 127.5 (d, $J 3, \mathrm{CH}), 127.0(\mathrm{~d}, J$ 90, C-1 of PPh), $126.0(\mathrm{~d}, J 9, \mathrm{CH}), 125.7(\mathrm{~d}, J 13,-\mathrm{CH}=), 123.7$ $(\mathrm{CH}), 123.5(\mathrm{CH}), 123.0(\mathrm{CH}), 71.4(\mathrm{~d}, J 109, \mathrm{C}=\mathrm{P}), 15.3(\mathrm{SMe})$ and $14.1(\mathrm{Me}) ; \delta_{\mathrm{P}}$ +15.7; m/z $548\left(\mathrm{M}^{+}, 31 \%\right), 533$ (15), 501 (100), 405 (7), 383 (5), 277 (7), 262 (35), 250 (9), 183 (26), 151 (7) and 108 (8).

\subsection{3 [(3-(3-Methyl-2-thienyl)propenoyl)(2-}

methoxyphenyl)methylene] triphenylphosphorane $\mathbf{3 4}$

This was prepared as in 2.5.2 using (2-methoxybenzyl)triphenylphosphonium bromide 30 ( $8.0 \mathrm{~g}, 17.0 \mathrm{mmol})$, butyllithium in hexanes $\left(6.8 \mathrm{~cm}^{3}, 2.5 \mathrm{M}, 17.0 \mathrm{mmol}\right)$ and 3-(3-methyl-2-thienyl)propenoyl chloride $33(1.61 \mathrm{~g}, 8.6 \mathrm{mmol})$ to give the product (4.16 g, 91\%) as bright orange prisms, mp $227-229^{\circ} \mathrm{C}$ (Found: $\mathrm{C}, 76.5 ; \mathrm{H}$, 5.25. $\mathrm{C}_{34} \mathrm{H}_{29} \mathrm{O}_{2} \mathrm{PS}$ requires C, 76.7; H, 5.5\%); $v_{\max } / \mathrm{cm}^{-1} 1620,1589,1495,1435$, $1359,1241,1201,1106,1073,1051,1027,961,854,802,747$ and $695 ; \delta_{\mathrm{H}} 7.70-7.49$ $(7 \mathrm{H}, \mathrm{m}, \mathrm{Ph}$ and vinyl-H), 7.48-7.25 (10H, m, 6'-H and $\mathrm{Ph}), 7.05(1 \mathrm{H}, \mathrm{t}, J$ 8, 4'-H), $6.93(1 \mathrm{H}, \mathrm{d}, J 7,5-\mathrm{H}), 6.80\left(1 \mathrm{H}, \mathrm{t}, J 8,5^{\prime}-\mathrm{H}\right), 6.70(1 \mathrm{H}, \mathrm{d}, J 7,4-\mathrm{H}), 6.63(1 \mathrm{H}, \mathrm{d}, J 16$, vinyl-H), $6.40\left(1 \mathrm{H}, \mathrm{d}, J 8,3^{\prime}-\mathrm{H}\right), 3.22(3 \mathrm{H}, \mathrm{s}, \mathrm{OMe})$ and $2.21(3 \mathrm{H}, \mathrm{s}, \mathrm{Me}) ; \delta_{\mathrm{C}} 179.6(\mathrm{~d}$, $J$ 5, CO), $158.9\left(\mathrm{~d}, J 3, \mathrm{MeOC}_{6} \mathrm{H}_{4} \mathrm{C}-2\right), 137.6(\mathrm{C}), 137.4(\mathrm{~d}, J 6, \mathrm{CH}), 136.7(\mathrm{CH})$, 133.8 (d, $J 10, \mathrm{C}-2$ of PPh), 131.4 (d, $J 2, \mathrm{C}-4$ of PPh), $130.9(\mathrm{C}), 128.3$ (d, $J 12, \mathrm{C}-3$ of PPh), 128.0 (d, $J 2, \mathrm{CH}), 127.3$ (d, $J$ 91, C-1 of PPh), 126.5 (d, $J 11, \mathrm{MeOC}_{6} \mathrm{H}_{4} \mathrm{C}-$ 
1), $125.7(\mathrm{~d}, J$ 12, $-\mathrm{CH}=), 125.6(\mathrm{CH}), 123.6(\mathrm{CH}), 120.3(\mathrm{CH}), 110.1(\mathrm{~d}, J$ 2, CH), $70.1(\mathrm{~d}, J 110, \mathrm{C}=\mathrm{P}), 54.3(\mathrm{OMe})$ and $14.1(\mathrm{Me}) ; \delta_{\mathrm{P}}+15.4 ; \mathrm{m} / \mathrm{z} 532\left(\mathrm{M}^{+}, 52 \%\right), 501$ (100), 367 (22), 277 (21), 262 (62), 201 (7), 183 (40), 165 (7) and 151 (14).

\subsection{4 [(3-(3-Methyl-2-thienyl)propenoyl)(2-trideuteriomethoxyphenyl)methyl- -ene] triphenylphosphorane $\mathbf{3 6}$ \\ This was prepared as in 2.5.2 using (2-} trideuteriomethoxybenzyl)triphenylphosphonium bromide 32 (2.0 g, $4.3 \mathrm{mmol})$, butyllithium in hexanes $\left(1.7 \mathrm{~cm}^{3}, 2.5 \mathrm{M}, 4.3 \mathrm{mmol}\right)$ and 3-(3-methyl-2thienyl)propenoyl chloride $33(0.41 \mathrm{~g}, 2.2 \mathrm{mmol})$ to give the product $(1.04 \mathrm{~g}, 88 \%)$ as bright yellow prisms, $\mathrm{mp} 227-230^{\circ} \mathrm{C} ; v_{\max } / \mathrm{cm}^{-1} 2065,1619,1495,1490,1376,1266$, $1245,1201,1100,747$ and $695 ; \delta_{\mathrm{D}} 3.24\left(\mathrm{OCD}_{3}\right) ; \delta_{\mathrm{H}} 7.64-7.52(7 \mathrm{H}, \mathrm{m}, \mathrm{Ph}$ and vinyl$\mathrm{H}), 7.48-7.26\left(10 \mathrm{H}, \mathrm{m}, 6^{\prime}-\mathrm{H}\right.$ and $\left.\mathrm{Ph}\right), 7.06\left(1 \mathrm{H}, \mathrm{t}, J 7,4^{\prime}-\mathrm{H}\right), 6.94(1 \mathrm{H}, \mathrm{d}, J 6,5-\mathrm{H})$, $6.81(1 \mathrm{H}, \mathrm{t}, J$ 5'-H), $6.71(1 \mathrm{H}, \mathrm{d}, J 6,4-\mathrm{H}), 6.63(1 \mathrm{H}, \mathrm{d}, J 16$, vinyl-H), $6.41(1 \mathrm{H}, \mathrm{d}, J$ $\left.7,3^{\prime}-\mathrm{H}\right)$ and $2.20(3 \mathrm{H}, \mathrm{s}, \mathrm{Me}) ; \delta_{\mathrm{C}}$ identical to 34 save for the absence of the signal at 54.3; $\delta_{\mathrm{P}}+15.4 ; \mathrm{m} / \mathrm{z} 535\left(\mathrm{M}^{+}, 48 \%\right), 501$ (100), 368 (20), 277 (15), 262 (69), 183 (58), $151(22)$ and 69 (77).

\subsection{Pyrolysis of Methylthienylacryloyl Ylides}

2.6.1 FVP of [(3-(3-methyl-2-thienyl)propenoyl(2-methoxyphenyl)methylene]triphenylphosphorane $\mathbf{3 4}$

FVP of the title compound $(0.5 \mathrm{~g})$ at $800{ }^{\circ} \mathrm{C}$ and $5.0-7.0 \times 10^{-2}$ Torr gave a brown oily solid at the furnace exit. Column chromatography of this using diethyl etherhexane (1:9) as eluant gave a yellow oil. GCMS, ${ }^{1} \mathrm{H}$ NMR, ${ }^{13} \mathrm{C}$ NMR and HRMS analysis indicated a mixture of three main products;

1. 5-benzylbenzothiophene 37 (63\%) (HRMS: Found $\mathrm{M}^{+}$224.0652. $\mathrm{C}_{15} \mathrm{H}_{12} \mathrm{~S}$ requires $M, 224.0660) ; \delta_{\mathrm{H}} 4.03\left(2 \mathrm{H}, \mathrm{s}, \mathrm{CH}_{2}\right) ; \delta_{\mathrm{C}} 41.8\left(\mathrm{CH}_{2}\right) ; m / z 224\left(\mathrm{M}^{+}, 99 \%\right), 206(10), 189$ (16), 179 (17), 147 (100), 111 (37) and 89 (72).

2. 5-( $\alpha$-methylbenzyl)benzothiophene 38 (21\%) (HRMS: Found $\mathrm{M}^{+} 238.0819$. $\mathrm{C}_{16} \mathrm{H}_{14} \mathrm{~S}$ requires $\left.M, 238.0816\right) ; \delta_{\mathrm{H}} 4.23(1 \mathrm{H}, \mathrm{q}, J 7, \mathrm{CH})$ and $1.64\left(3 \mathrm{H}, \mathrm{d}, J 7, \mathrm{CH}_{3}\right)$; $\delta_{\mathrm{C}} 44.7(\mathrm{CH})$ and $22.1\left(\mathrm{CH}_{3}\right) ; \mathrm{m} / z 238\left(\mathrm{M}^{+}, 44 \%\right), 223(100), 208(10), 189(22), 179$ (25), $111(51), 89$ (50) and 77 (58).

3. fluoreno [3,4-b] thiophene 39 (16\%) (HRMS: Found $\mathrm{M}^{+}$222.0512. $\mathrm{C}_{15} \mathrm{H}_{10} \mathrm{~S}$ requires $M, 222.0503) ; \delta_{\mathrm{H}} 3.90\left(2 \mathrm{H}, \mathrm{s}, \mathrm{CH}_{2}\right) ; \delta_{\mathrm{C}} 37.2\left(\mathrm{CH}_{2}\right) ; m / z 222\left(\mathrm{M}^{+}, 100 \%\right), 189(23)$, $176(17), 150(9), 111(73)$ and $88(23)$.

\subsubsection{FVP of [(3-(3-methyl-2-thienyl)propenoyl)(2-methylthiophenyl)methyl- ene] triphenylphosphorane $\mathbf{3 5}$}

FVP of the title compound $(0.5 \mathrm{~g})$ at $800{ }^{\circ} \mathrm{C}$ and $2.0-7.0 \times 10^{-2}$ Torr gave a brown oily solid at the furnace exit. Column chromatography of this using diethyl etherhexane (1:9) as eluant gave a yellow oil. GCMS, ${ }^{1} \mathrm{H}$ NMR, and ${ }^{13} \mathrm{C}$ NMR analysis suggested a mixture of three main products:

1. 5-benzylbenzothiophene $37(52 \%) ; \delta_{\mathrm{H}} 4.01\left(2 \mathrm{H}, \mathrm{s}, \mathrm{CH}_{2}\right) ; \delta_{\mathrm{C}} 41.8\left(\mathrm{CH}_{2}\right) ; m / z 224$ $\left(\mathrm{M}^{+}, 95 \%\right), 208(8), 189(16), 179$ (18), $147(100), 111(40)$ and 89 (68).

2. 5-( $\alpha$-methylbenzyl)benzothiophene $38(23 \%) ; \delta_{\mathrm{H}} 4.19(1 \mathrm{H}, \mathrm{q}, J 7, \mathrm{CH})$ and 1.62 $\left(3 \mathrm{H}, \mathrm{d}, J 7, \mathrm{CH}_{3}\right) ; \delta_{\mathrm{C}} 44.6(\mathrm{CH})$ and $22.0\left(\mathrm{CH}_{3}\right) ; \mathrm{m} / z 238\left(\mathrm{M}^{+}, 42 \%\right), 223(100), 208$ (9), 189 (19), 179 (26), 111 (67), 89 (72) and 77 (83). 
3. fluoreno[3,4-b]thiophene $39(25 \%) ; \delta_{\mathrm{H}} 3.82\left(2 \mathrm{H}, \mathrm{s}, \mathrm{CH}_{2}\right) ; \delta_{\mathrm{C}} 37.1\left(\mathrm{CH}_{2}\right) ; m / z 222$ $\left(\mathrm{M}^{+}, 90 \%\right), 189$ (24), $176(18), 150$ (12), 110 (100), 98 (39) and $88(40)$.

2.6.3 FVP of [(3-(3-methyl-2-thienyl)propenoyl)(2-trideuteriomethoxyphenyl)methylene] triphenylphosphorane $\mathbf{3 6}$

FVP of the title compound $(0.5 \mathrm{~g})$ at $800{ }^{\circ} \mathrm{C}$ and $2.0-5.0 \times 10^{-2}$ Torr gave a brown oily solid at the furnace exit. Column chromatography of this using diethyl etherhexane (1:9) as eluant gave a yellow oil. GCMS, ${ }^{1} \mathrm{H}$ NMR, ${ }^{2} \mathrm{H}$ NMR and ${ }^{13} \mathrm{C}$ NMR analysis indicated a mixture of four products;

1. 5-benzylbenzothiophene $37 \delta_{\mathrm{H}} 4.01(2 \mathrm{H}, \mathrm{s}, \mathrm{CH} 2) ; \delta_{\mathrm{C}} 41.8\left(\mathrm{CH}_{2}\right) ; \mathrm{m} / z 224\left(\mathrm{M}^{+}\right.$, $100 \%)$.

2. 5-( $\alpha$-deuteriobenzyl)benzothiophene $40 \delta_{\mathrm{H}} 4.00(1 \mathrm{H}, \mathrm{s}, \mathrm{CHD}) ; \delta_{\mathrm{D}} 4.18(1 \mathrm{D}, \mathrm{d}, J 2$, CHD); $\delta_{\mathrm{C}} 41.5$ (t, J 20, CHD); $m / z 225$ (M $\mathrm{M}^{+}, 100 \%$ ).

3. 5-( $\alpha$-trideuteriomethylbenzyl)benzothiophene $41 \delta_{\mathrm{H}} 4.18(1 \mathrm{H}, \mathrm{CH}) ; \delta_{\mathrm{D}} 1.79(3 \mathrm{D}, \mathrm{s}$, $\left.\mathrm{CD}_{3}\right) ; \delta_{\mathrm{C}} 44.4(\mathrm{CH}) ; m / z 241\left(\mathrm{M}^{+}, 33 \%\right), 223(100)$.

4. fluoreno[3,4-b]thiophene $39 \delta_{\mathrm{H}} 3.87\left(2 \mathrm{H}, \mathrm{CH}_{2}\right) ; \delta_{\mathrm{C}} 37.2\left(\mathrm{CH}_{2}\right) ; m / z 222\left(\mathrm{M}^{+}\right.$, $100 \%)$.

\section{$3 \quad$ Results and discussion}

\subsection{Synthesis and FVP of simple methoxythenoyl ylides}

A series of methoxythenoyl ylides 8-13 were readily prepared by acylation of either benzylidene- or 1-propylidene-triphenylphosphorane in THF with the appropriate methoxythenoyl chlorides (Scheme 3). While 3-methoxy-2-thenoyl chloride required for 8 and 9 [21] and 2-methoxy-3-thenoyl chloride required for 13 [22] have been previously described, 4-methoxy-3-thenoyl chloride required for $\mathbf{1 0}$ and $\mathbf{1 2}$, as well as its deuterium-labelled analogue required for 11, have not. They were prepared by treatment of the corresponding 4-methoxythiophene-3-carboxylic acids with thionyl chloride and obtained as low melting solids.
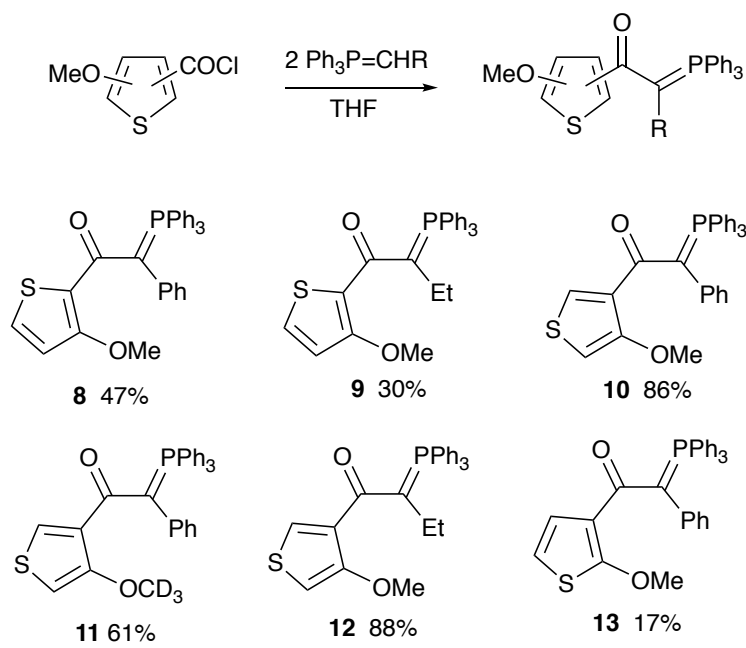

Scheme 3. Simple methoxythenoyl ylides prepared

The ylides 8-13 were obtained as high-melting crystalline solids which were stable on storage and showed the expected spectroscopic properties including ${ }^{31} \mathrm{P}$ NMR signals in the range $+15.6-17.3 \mathrm{ppm}$ and ${ }^{13} \mathrm{C}$ NMR spectra with phosphorus coupling 
extending throughout the $P$-phenyl groups, the oxo ylide function and to at least the first carbon of both $\mathrm{Ph} / \mathrm{Et}$ and thienyl groups. Importantly for the planned extrusion of $\mathrm{Ph}_{3} \mathrm{PO}$ under FVP conditions, the value of the phosphorus coupling to the carbonyl group, ${ }^{2} J_{\mathrm{p}-\mathrm{CO}}$ was $6-8 \mathrm{~Hz}$, meaning that the extrusion should proceed readily since we recently showed [28] an empirical correlation with a cut off of $10 \mathrm{~Hz}$.

The expected process upon FVP of ylides 8 and 9 (Scheme 4) involves cyclisation of the alkyne-containing thienyloxy radical to give the thieno[3,2-b]furyl radical which then gives an isolable product in which the substituent may be the same or different. According to our previous work [29,30], ylides with $\mathrm{R}=\mathrm{Ph}$ should give the furan product with $\mathrm{R}^{1}=\mathrm{Ph}$ by simple hydrogen atom abstraction from the environment, while for $\mathrm{R}=\mathrm{Et}$, the main product should be $\mathrm{R}^{1}=$ vinyl formed by intramolecular hydrogen atom abstraction and loss of $\mathrm{H}$.

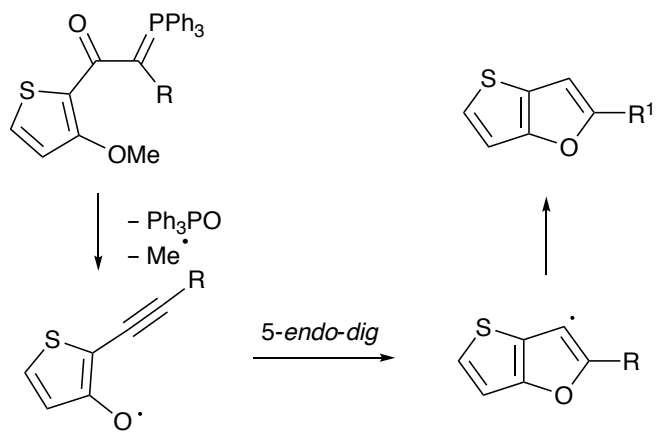

Scheme 4. Expected mechanism of formation of thieno[3,2-b]furans

For the ylides 8 and 9 and also the isomeric system 12, FVP at $800{ }^{\circ} \mathrm{C}$ resulted in complete reaction to give, after chromatographic separation of $\mathrm{Ph}_{3} \mathrm{PO}$, heterocyclic products with the expected thienofuran structures (Scheme 5). In the case of $\mathbf{8}$, the product was fully characterised as 2-phenylthieno[3,2-b]furan 14 formed in moderate yield. It should be noted that there is a single literature report of this compound [31], but the spectroscopic data given there appears to be erroneous, particularly the absence of the expected two ${ }^{13} \mathrm{C}$ NMR signals above $150 \mathrm{ppm}$ for the quaternary carbons directly attached to oxygen which for our product appeared at 158.0 and $157.8 \mathrm{ppm}$.
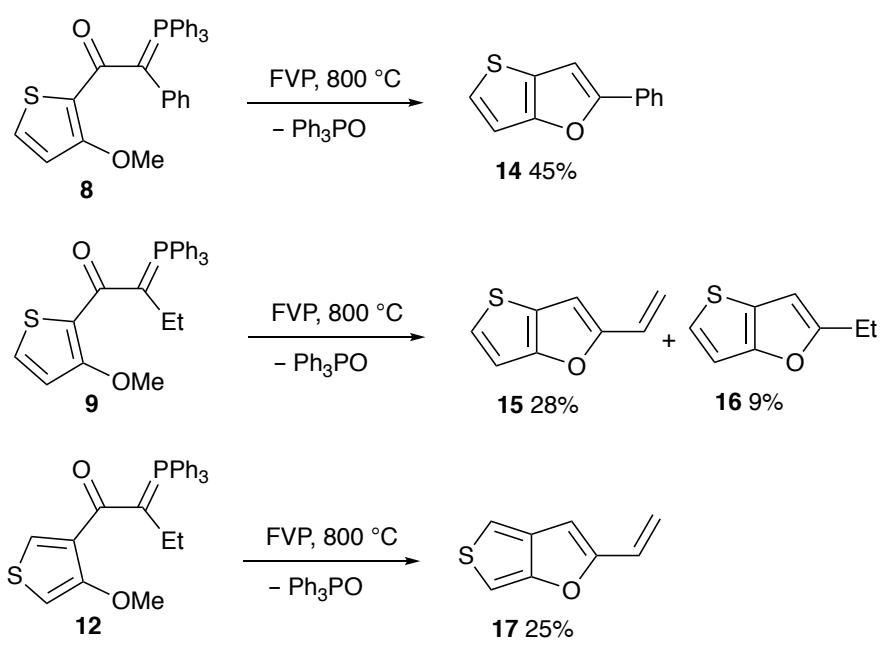

Scheme 5. FVP of ylides $\mathbf{8 , 9}$ and $\mathbf{1 2}$ to give thienofuran products 
For the ethyl ylide 9, the product was obtained as an oil consisting of a mixture of components but the major product was identified spectroscopically as the expected 2vinylthieno[3,2-b]furan $\mathbf{1 5}$ accompanied by a little of the corresponding ethyl compound 16 formed by hydrogen atom abstraction by the intermediate radical. In the case of the isomeric system 12, chromatographic separation followed by vacuum distillation gave 2-vinylthieno[3,4- $b]$ furan in pure form as an unstable liquid.

To our surprise, the phenyl ylide $\mathbf{1 0}$ with the structure analogous to 12, behaved in a completely different way. Initial FVP at $800{ }^{\circ} \mathrm{C}$ led to formation of $\mathrm{Ph}_{3} \mathrm{PO}$ but the other products were clearly not the expected thienofuran and could not be immediately identified. For this reason, FVP at the lower temperature of $725^{\circ} \mathrm{C}$ was examined and the deuterium labelled analogue $\mathbf{1 1}$ was also prepared. Chromatographic separation of the product mixture obtained at $725{ }^{\circ} \mathrm{C}$ (Scheme 6) gave the alkyne 20 expected from extrusion of $\mathrm{Ph}_{3} \mathrm{PO}$, together with 2-phenyl-3vinylthiophene 18 and 1-phenylbuta-1,3-diyne 19. The identity of the diyne was confirmed by excellent agreement of both ${ }^{1} \mathrm{H}$ and ${ }^{13} \mathrm{C}$ NMR data with reported values [23]. The isolation of alkyne $\mathbf{2 0}$ and the fact that it was converted into $\mathbf{1 8}$ and $\mathbf{1 9}$ upon being re-subjected to FVP at $800{ }^{\circ} \mathrm{C}$ strongly suggest that the first stage of the pyrolytic process is proceeding as expected and that compounds $\mathbf{1 8}$ and $\mathbf{1 9}$ are formed as a result of secondary pyrolytic breakdown of $\mathbf{2 0 .}$

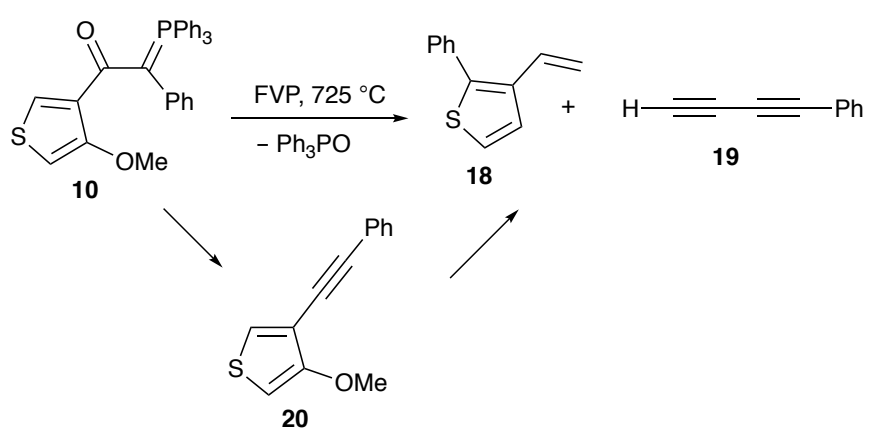

Scheme 6. Anomalous behaviour of ylide $\mathbf{1 0}$ upon FVP

It was therefore of great interest to examine FVP of the deuterium-labelled ylide $\mathbf{1 1}$. At $725{ }^{\circ} \mathrm{C}$ it gave the labelled alkyne 21 together with unlabelled phenylbutadiyne $\mathbf{1 9}$ and 2-phenyl-3-vinylthiophene $\mathbf{2 2}$ bearing two deuterium atoms on the terminal position of the vinyl group and a third on position 4 of the thiophene ring (Scheme 7). As for the unlabelled pyrolysis mixture, re-pyrolysis at $800{ }^{\circ} \mathrm{C}$ led to complete conversion of $\mathbf{2 1}$ into $\mathbf{1 9}$ and 22.

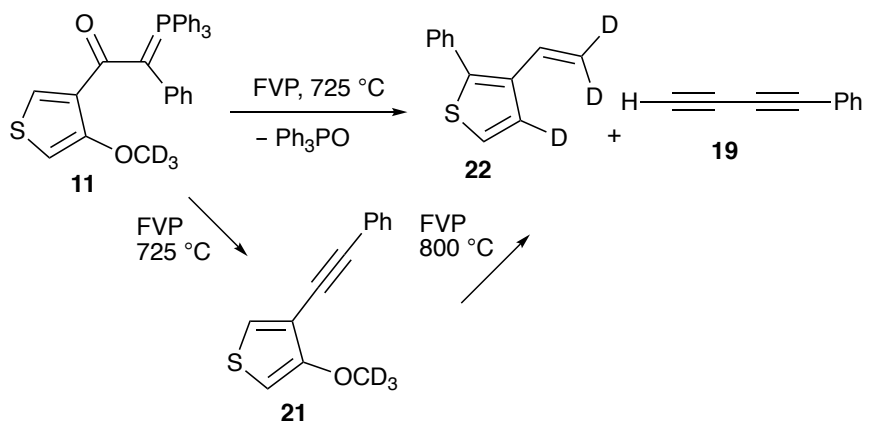

Scheme 7. Result of FVP of the deuterium-labelled ylide 11 
Based on these results, and particularly the fact that $\mathbf{1 9}$ is formed in unlabelled form, we believe that the diyne results from fragmentation of $\mathbf{2 1}$ (or 20) as shown in Scheme 8 .
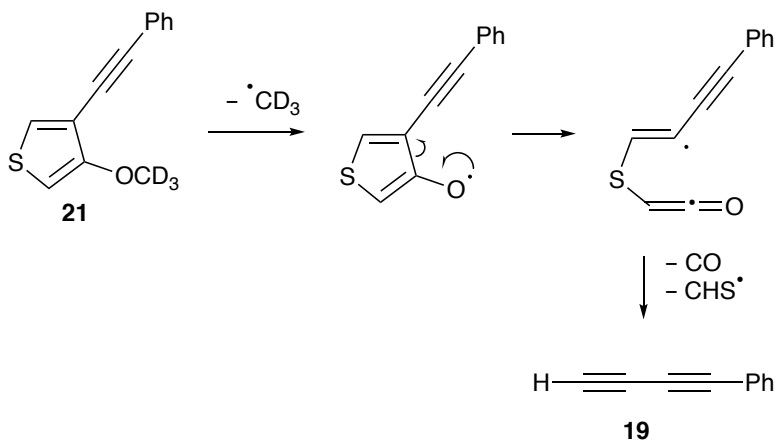

Scheme 8. Suggested mechanism for formation of diyne 19

The formation of $\mathbf{1 8}$ is more surprising and harder to explain but a mechanism that is consistent with the deuterium labelling pattern of $\mathbf{2 2}$ is shown in Scheme 9. The key feature of this is isomerisation of the alkyne function in $\mathbf{2 1}$ (or 20) to a vinylidene, a well precedented process under these conditions [32], and then triplet reactivity of this leading to a series of radical abstraction and rearrangement steps.
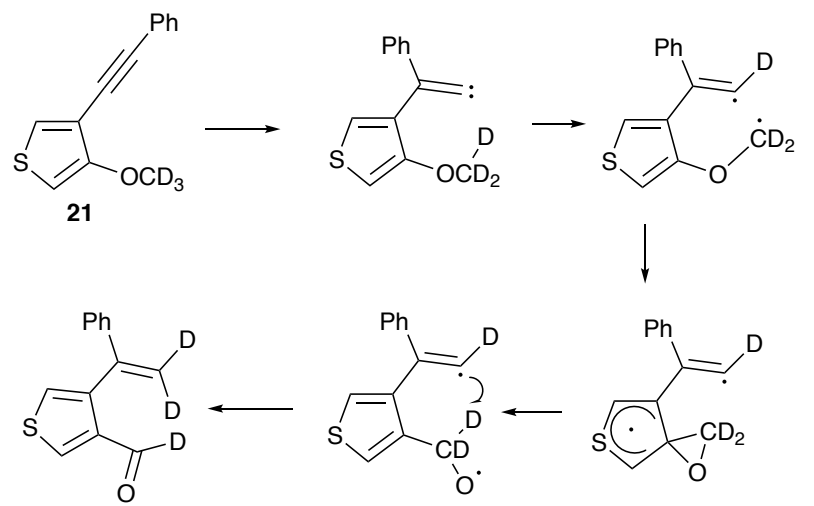<smiles>C1C[Te]C1</smiles><smiles>[2H]C([2H])=Cc1c([2H])csc1-c1ccccc1</smiles>

Scheme 9. Proposed mechanism for formation of labelled vinylthiophene $\mathbf{2 2}$

Finally, in this section, FVP of the ylide 13, isomeric with $\mathbf{8}$ and $\mathbf{1 0}$ did not lead to any useful products. There was extensive decomposition at $75^{\circ} \mathrm{C}$ to give products including $\mathrm{Ph}_{3} \mathrm{PO}, \mathrm{Ph}_{3} \mathrm{P}$ and $\mathrm{Ph}_{3} \mathrm{PS}$ together with biphenyl and dibenzothiophene.

\subsection{Synthesis and FVP of methoxythienyl cinnamoyl ylides}

Based on the encouraging cyclisation behaviour observed for ylides $\mathbf{8}$ and $\mathbf{9}$ and to a lesser extent 10 and 12, synthesis of extended analogues capable of domino 
cyclisation to give polycyclic products was now planned. Since $\mathbf{1 3}$ did not give good results, no extended ylides with the 2-methoxy-3-thienyl structure were examined.

In contrast to the simple ylides which could be prepared in one step from a commercial phosphonium salt and a thiophene-containing acid chloride, the extended ylides required the methoxythiophene group to be located in the phosphonium salt and thus involved multi-stage synthesis (Scheme 10). As we noted some time ago [24], attempted lithium aluminium hydride reduction of methoxythiophene esters is not without complication. Any contact of compounds such as $\mathbf{2 3}$ with acid leads to oligomerisation so completely non-acidic conditions must be used. The readily available methyl 3-methoxythiophene-2-carboxylate [25] was thus reduced with a neutral/basic work-up [24] to give $\mathbf{2 3}$ and this was followed by combined chlorination and phosphonium salt formation, also under non-acidic conditions using $\mathrm{Ph}_{3} \mathrm{P} / \mathrm{CCl}_{4}$. The resulting phosphonium salt $\mathbf{2 4}$ was then deprotonated and acylated with 0.5 equiv. of either cinnamoyl chloride or the thiophene-containing analogue [26] to give, respectively, ylides $\mathbf{2 5}$ and $\mathbf{2 6}$.

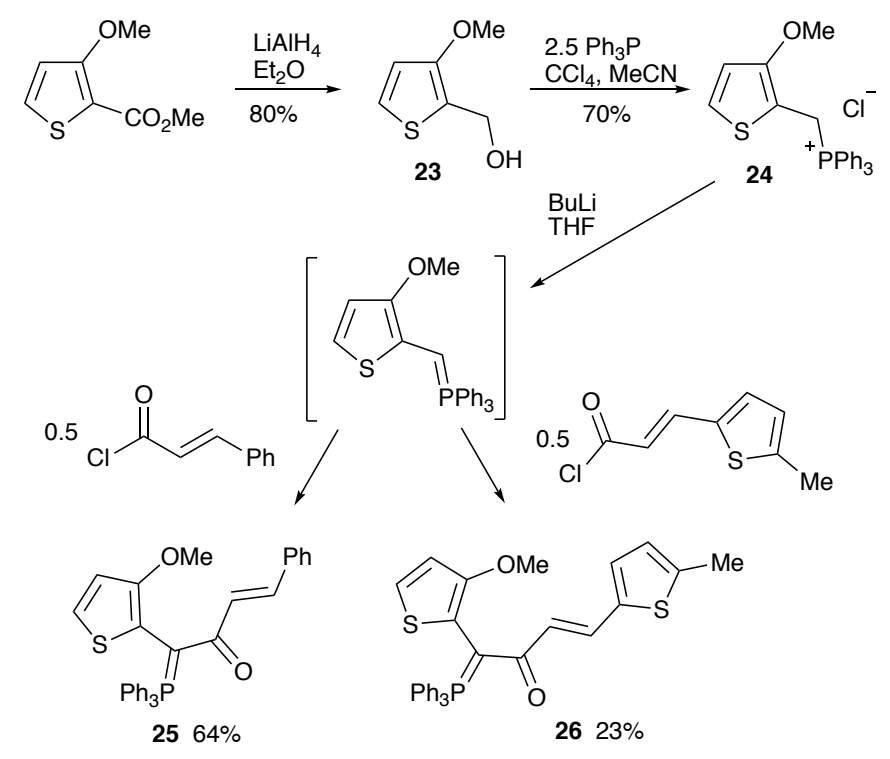

Scheme 10. Synthesis of extended methoxythienyl ylides $\mathbf{2 5}$ and 26

The ylide 29 with the isomeric structure was likewise prepared (Scheme 11) starting from methyl 4-methoxythiophene-3-carboxylate [20] by $\mathrm{LiAlH}_{4}$ reduction under nonacidic conditions [24] to give 27, chlorination and phosphonium salt formation to give 28 followed by deprotonation and treatment with 0.5 equiv. cinnamoyl chloride. The three ylides 25, 26 and 29 were obtained as high-melting stable crystalline solids with values of ${ }^{2} J_{\mathrm{P}-\mathrm{CO}}$ of $8-9 \mathrm{~Hz}$ leading us to predict [28] successful extrusion of $\mathrm{Ph}_{3} \mathrm{PO}$ upon FVP. 

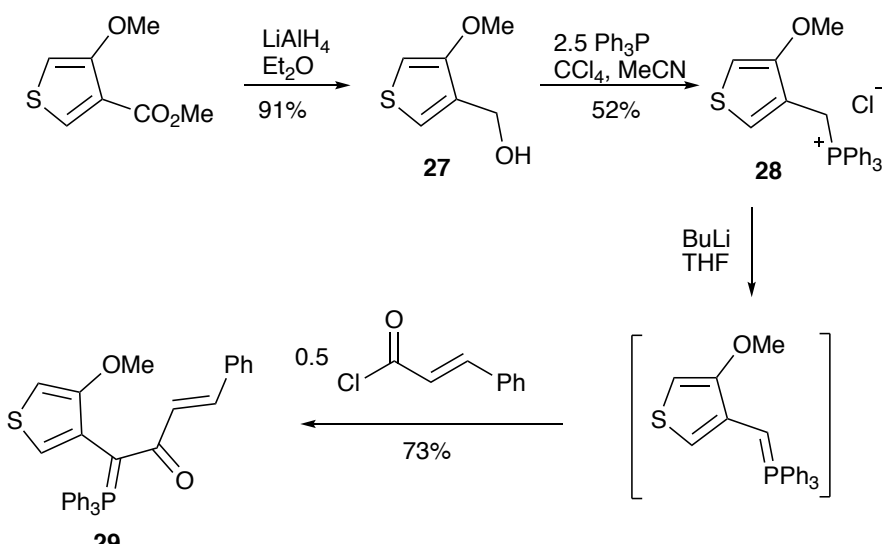

Scheme 11. Synthesis of extended methoxythienyl ylide 29

Unfortunately, however no useful products were obtained from FVP of any of the three ylides. In each case there was extensive decomposition in the range $700-750{ }^{\circ} \mathrm{C}$ with no identifiable heterocyclic products obtained.

\subsection{Synthesis and FVP of methoxythienylacryloyl ylides}

In a previous study, ylides bearing a 2-methylcinnamoyl group were found to give rise to naphthalenes upon FVP in a process involving incorporation of the methyl group into a benzene ring at the stage of the 2-methylstyrylalkyne [33,34]. In a single example the same reaction was also observed for a thiophene analogue. We wanted to see whether this behaviour could be combined with benzofuran or benzothiophene formation in a single domino cyclisation process and so designed the ylides $\mathbf{3 4}$ and $\mathbf{3 5}$ containing both the required features. The new acid chloride $\mathbf{3 3}$ was first prepared by treating the corresponding acrylic acid [27] with oxalyl chloride and fully characterised. This was then used to acylate the ylides derived by treatment of phosphonium salts 30 and $\mathbf{3 1}$ with butyllithium to give $\mathbf{3 4}$ and $\mathbf{3 5}$ respectively Scheme 12). In order to elucidate the unexpected pyrolysis behaviour, the deuterium-labelled salt 32 was similarly used to generate the labelled ylide 36. All three ylides were formed in excellent yield as stable solids and the ${ }^{2} J_{\mathrm{P}-\mathrm{CO}}$ values of $5-6 \mathrm{~Hz}$ again suggested successful thermal extrusion of $\mathrm{Ph}_{3} \mathrm{PO}$ [28].

2<smiles>[X]c1ccccc1C[Pb]Br</smiles>

$30 \mathrm{X}=\mathrm{OMe}$ $31 X=S M e$ $32 \mathrm{X}=\mathrm{OCD}_{3}$

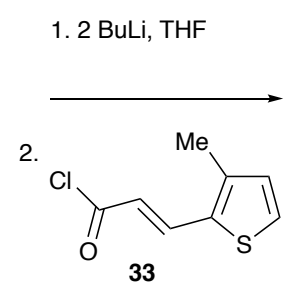

Scheme 12. Synthesis of 3-methyl-2-thienylacryloyl ylides 34-36

In the event, FVP of both the OMe ylide 34 and the SMe ylide 35 led to complete reaction at $800{ }^{\circ} \mathrm{C}$ and, most surprisingly, the products were the same in each case (Scheme 13). Chromatographic separation of $\mathrm{Ph}_{3} \mathrm{PO}$ left a mixture containing 5benzylbenzothiophene 37, 5-( $\alpha$-methylbenzyl)benzothiophene 38 and fluoreno[3,4$b]$ thiophene 39. 


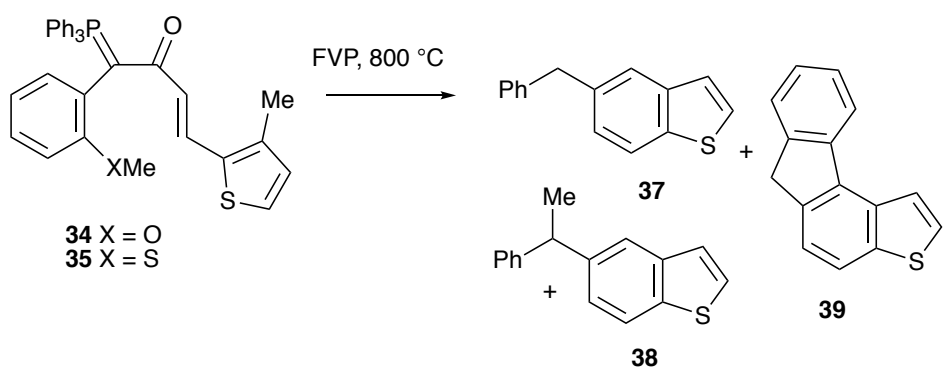

Scheme 13. FVP of ylides 34 and 35

Interestingly two of these products, $\mathbf{3 7}$ and $\mathbf{3 9}$ were formed in our previous work [34] by FVP of the ylide with the same structure as $\mathbf{3 4}$ or $\mathbf{3 5}$ but with XMe replaced by $\mathrm{H}$. The spectroscopic data obtained in the two studies were identical. Since, in addition to explaining the formation of $\mathbf{3 7}$ and $\mathbf{3 9}$ here, we have to explain the disappearance of the XMe group and also the appearance of the extra methyl group in product $\mathbf{3 8}$ the FVP result starting with the deuterium-labelled ylide $\mathbf{3 6}$ was of great interest.
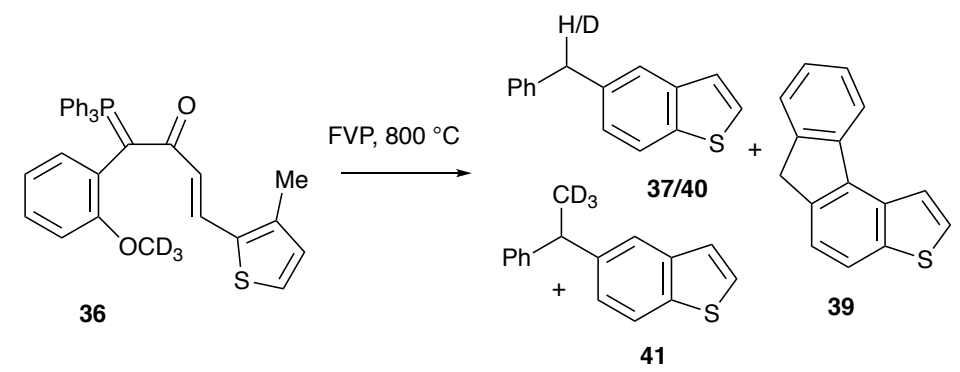

Scheme 14. FVP of deuterium-labelled ylide 36

When this was performed, it gave a clear result (Scheme 14): the same three products were formed as for the unlabelled compound and by a combination of ${ }^{1} \mathrm{H},{ }^{2} \mathrm{H}$ and ${ }^{13} \mathrm{C}$ NMR and GCMS analysis, it was determined that 5-benzylbenzothiophene 37 was partly monodeuterated at the benzylic position (40), the tetracyclic product 39 was unlabelled and the 5-( $\alpha$-methylbenzyl)benzothiophene was fully trideuterated, i.e. 41.

Our proposal for the mechanisms involved which is supported by the deuterium-labelling results (Scheme 15) starts with extrusion of $\mathrm{Ph}_{3} \mathrm{PO}$ and formation of a thienylmethyl radical which can undergo cyclisation to form $\mathbf{4 2}$ already containing the carbon skeleton required for products 37 and 39. However, in contrast to the previous study, this intermediate now has the OMe/SMe group ideally placed to suffer an intramolecular hydrogen atom abstraction, thus setting in motion a series of steps that ultimately results in loss of the $\mathrm{XMe}$ group most likely as $\mathrm{CH}_{2} \mathrm{X}$, either formaldehyde or thioformaldehyde. The possibility of 1,3-hydrogen shifts may be used to explain the partial loss of the deuterium atom from the benzylic position. It should be noted that such loss of a methoxy group to be replaced by hydrogen was previously observed in our earlier studies on benzofuran and benzothiophene formation [9]. Once this group is lost formation of products $\mathbf{3 7}$ and $\mathbf{3 9}$ is readily explained. The only reasonable source of the methyl group in product $\mathbf{3 8}$ is from the original OMe or SMe group of the ylides and this was confirmed by the formation of the trideuteriomethyl compound $\mathbf{4 1}$ from ylide 36. Although such an intramolecular process seems inherently unlikely in the gas phase, it has good precedent in this area of work with the formation of benzofuran bearing a $2-\mathrm{CH}_{2} \mathrm{CD}_{3}$ group from a range of 
$\mathrm{OCD}_{3}$-containing ylides that gave rise to a benzofurylmethyl radical intermediate [29, 30]. Once the new methyl group has been attached, reactivity is restored to the benzylic centre by formation of a stable radical in order to promote the loss of the adjacent XMe group as before to produce $\mathbf{3 8}$.

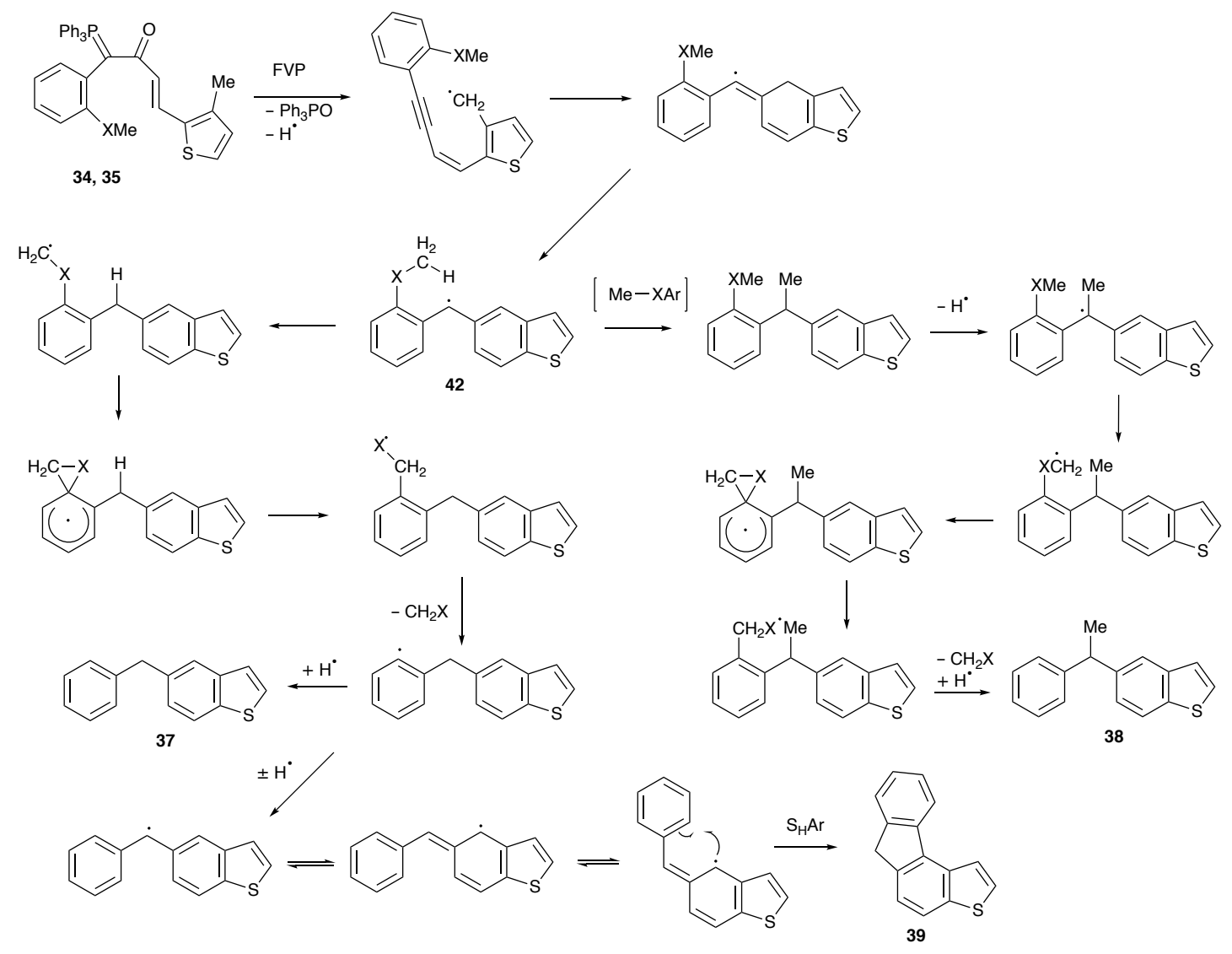

Scheme 15. Proposed mechanism for FVP of $\mathbf{3 4}$ and $\mathbf{3 5}$ to give $\mathbf{3 7}, \mathbf{3 8}$ and $\mathbf{3 9}$

\section{Conclusion}

While the studies on FVP of simpler methoxythienyl ylides 8, 9 and 12 led to successful formation of the expected thienofuran products, the approach clearly has limitations with the isomeric ylide $\mathbf{1 3}$ affording no fused ring product and ylide $\mathbf{1 0}$ closely related to $\mathbf{1 2}$ giving a completely different and unexpected result which is nonetheless of significant mechanistic interest. Attempts to capitalise on these promising findings by moving to more extended ylides such as 25, 26 and 29 unfortunately met with failure. The ylides were successfully prepared and characterised but gave no useful products upon FVP probably due to the increased fragility of their structures under the relatively harsh conditions required for reaction. The two ylides $\mathbf{3 4}$ and 35, where two competing thermal cyclisation processes are possible, unexpectedly underwent benzothiophene formation based on cyclisation of the thienylmethyl group with complete loss of the normal cyclisation-initiating groups $\mathrm{OMe}$ and $\mathrm{SMe}$, thus giving some idea of the relative ease of these two competing processes. 


\section{Acknowledgements}

We thank EPSRC (UK) for a studentship to ANG.

\section{References}

[1] C. Wentrup, Flash vacuum pyrolysis: techniques and reactions, Angew. Chem. Int. Ed. 56 (2017) 14808-14835.

[2] R.A. Aitken, Y. Boubalouta, Recent advances in the synthesis of heterocyclic compounds using flash vacuum pyrolysis, Adv. Heterocycl. Chem., E.F.V. Scriven, C.A. Ramsden (Eds.) 115 (2015) 93-150.

[3] R.A. Aitken, A.N. Garnett, Versatile pyrolytic synthesis of fused polycyclic heteroaromatic compounds, Synthesis 49 (2017) 4955-4977.

[4] R.A. Aitken, A.N. Garnett, An eight-stage gas-phase cascade reaction leading to 7-(2-benzothienyl)benzofuran and 2,7'-bi(benzofuran), Synlett (2001) 228-229.

[5] R.A. Aitken, A.N. Garnett, Cascade synthesis of new tetracyclic heteroaromatic thieno[2,3-b]pyridine-containing ring systems, New J. Chem. 33 (2009) 24022404.

[6] R.A. Aitken, L. Murray, New gas-phase cascade reactions of stabilized phosphorus ylides leading to ring-fused indoles and to quinolines, J. Org. Chem. 73 (2008) 9781-9783.

[7] R.A. Aitken, L. Murray, Gas-phase domino cyclization of phosphonium ylides leading to the total synthesis of Eustifoline D, Tetrahedron Lett. 58 (2017) 4328-4332.

[8] R.A. Aitken, L. Murray, A.M.Z. Slawin, Further studies on the pyrolytic domino cyclization of stabilized ylides bearing an ortho-aminophenyl group, Molecules (2018)-.

[9] R.A. Aitken, G. Burns, J.J. Morrison, Flash vacuum pyrolysis of stabilised phosphorus ylides. Part 14. Tandem cyclisation of intermediate aryloxy and arylthio radicals leading to tri- and tetra-cyclic aromatic heterocycles, J. Chem. Soc., Perkin Trans. 1 (1998) 3937-3941.

[10] S.P. Stanforth, Five-five fused hetarenes with one heteroatom in each ring, Science of Synthesis 10.21 (2014), 1-148.

[11] N. Hergué, C. Mallet, J. Touvron, M. Allain, P. Leriche, P. Frère, Facile synthesis of 3 -substituted thieno[3,2-b]furan derivatives, Tetrahedron Lett. 49 (2008) 2425-2428.

[12] J.T. Henssler, A.J. Matzger, Facile and scalable synthesis of the fused-ring heterocycles thieno[3,2-b] thiophene and thieno[3,2-b]furan, Org. Lett. 11 (2009) 3144-3147.

[13] V.Z. Shirinian, A.A. Shimkin, S.N. Tipikin, M.M. Krayushkin, Efficient methods for the synthesis of thieno[3,2-b]thiophene and thieno[3,2-b]furan derivatives, Synthesis (2009) 3803-3806.

[14] C. Gadais, S. Hesse, G. Kirsch, Regioselective Thorpe-Ziegler cyclization of 3$O$-alkylated thiophenes; access to aminothieno[3,2-b]furans and aminothieno[3,4-b]furans, Syntheis (2012) 2964-2968.

[15] A Shafiee, M.A. Ebrahimzadeh, J. Shahbazi, S. Hamedpanah, Synthesis of $1 \mathrm{H}-$ thieno[3,4-c]pyrazoles, thieno[3,4-b]furans, seleno[3,4-b]furans and pyrrolo[3,4-d] thiazoles, J. Heterocycl. Chem. 35 (1998) 71-75. 
[16] M.R. Banks, J.M. Barker, P.R. Huddleston, Preparation and some reactions of substituted thieno[3,4-b]furans, J. Chem. Soc., Perkin Trans. 1 (1986) 22232232

[17] R.A. Aitken, A.O. Oyewale, Unexpected rearrangement during the gas-phase dehalogenation approach to benzodithiophenes, RSC Advances, 5 (2015) 19379-19381.

[18] R.A. Aitken, J. I. Atherton, Flash vacuum pyrolysis of stabilised phosphorus ylides. Part 1. Preparation of aliphatic and terminal alkynes, J. Chem. Soc., Perkin Trans. 1 (1994) 1281-1284.

[19] R.A. Aitken, C.E.R. Horsburgh, Flash vacuum pyrolysis of $o$-phenylene sulfite: formation and purification of cyclopentadienone dimer. In Comprehensive Organic Chemistry Experiments for the Laboratory Classroom; C.A.M. Afonso, N.R. Candeias, D.P. Simao, A.F. Trinidade, J.A.S. Coelho, B. Tan, R. Franzén, Eds; Royal Society of Chemistry: Cambridge, UK, 2017; Section 10.9, pp. 690693.

[20] J.B. Press, C.M. Hofmann, S.R. Safir, Thiophene systems 2. Synthesis and chemistry of some 4-alkoxy-3-substituted thiophene derivatives, J. Org. Chem. 44 (1979) 3292-3296.

[21] G. Henrio, J. Morei, P. Pastour, Sur la synthese d'isosteres thiopheniques des flavones, thioflavones et xanthones, Tetrahedron 33 (1977) 191-198.

[22] A. Hallberg, S. Gronowitz, On the Vilsmeier formylation of some methoxythiophenes, Chemica Scripta 16 (1980) 38-41.

[23] M.X.-W. Jiang, M. Rawat, W.D. Wulff, Contingency and serendipity in the reactions of Fischer carbene complexes with conjugated triynes, J. Am. Chem. Soc 126 (2004) 5970-5971.

[24] R.A. Aitken, A.N. Garnett, A new reaction of electron-rich thiophenemethanols, J. Chem. Soc., Perkin Trans. 1 (2000) 3020-3021.

[25] H. Fiesselmann, P. Schipprak, L. Zeitler, Über Oxythiophen-carbonsäureester, II Mitteil.: Synthese und Reaktionen von 3-Oxy-thiophen-carbonsäure-(2)-estern, Chem. Ber. 87 (1954) 841-848.

[26] R.A. Aitken, C. Boeters, J.J. Morrison, Flash vacuum pyrolysis of stabilised phosphorus ylides. Part 6. Pyrolysis of substituted cinnamoyl ylides as a route to conjugated enynes, J. Chem. Soc., Perkin Trans. 1 (1994) 2473-2480.

[27] W.J. King, F.F. Nord, Studies in the thiophene series, III. Condensations of thiophenealdehydes, J. Org. Chem. 14 (1949) 405-410.

[28] R.A. Aitken, Y. Boubalouta, D. Chang, L.P. Cleghorn, I.P. Gray, N. Karodia, E.J. Reid, A.M.Z. Slawin, The value of ${ }^{2} J_{\mathrm{P}-\mathrm{CO}}$ as a diagnostic parameter for the structure and thermal reactivity of carbonyl-stabilised phosphonium ylides, Tetrahedron 73 (2017) 6275-6285.

[29] R.A. Aitken, G. Burns, A new synthesis of 2-substituted benzofurans and benzothiophens: Novel fragmentation reactions of simple alkyl groups, Tetrahedron Lett. 28 (1987) 3717-3718.

[30] R.A. Aitken, G. Burns, Flash vacuum pyrolysis of stabilised phosphorus ylides. Part 3. Preparation of $o$-methoxyphenyl- and $o$-methylsulfanylphenyl-alkynes and their cyclisation to benzofurans and benzothiophenes, J. Chem. Soc., Perkin Trans. 1 (1994) 2455-2460.

[31] C.B. Lavery, N.L. Rotta-Loria, R. McDonald, M. Stradiotto, $\mathrm{Pd}_{2} \mathrm{dba}_{3} /$ bippyphos: A robust catalyst system for the hydroxylation of aryl halides with broad substrate scope, Adv. Synth. Catal. 355 (2013) 981-987. 
[32] R.F.C. Brown, Thermal rearrangments of alkynes under FVP conditions - the acetylene methylenecarbene rearrangement, Recl. Trav. Chim. Pays-Bas 107 (1988) 655-661.

[33] R A. Aitken, C. Boeters, J.J. Morrison, Novel thermal cyclisation of omethylstyrylalkynes to give 2-alkenylnaphthalenes, Tetrahedron Lett. 36 (1995) 1303-1306.

[34] R.A. Aitken, C. Boeters, J.J. Morrison, Flash vacuum pyrolysis of stabilized phosphorus ylides. Part 10. Generation of 2-methylstyrylalkynes and their thermal cyclisation to 2-alkenylnaphthalenes, J. Chem. Soc., Perkin Trans. 1 (1997) 2625-2631. 


\section{Highlights}

- Ten new oxo-stabilised phosphonium ylides are prepared

- FVP of simple examples gives thieno[3,2-b]furan and thieno[3,4-b]furan products

- Unexpected fragmentation and rearrangement occurs under milder conditions in one case to give phenylbutadiyne and a phenylvinylthiophene

- FVP of 3-methyl-2-thienylacryloyl ylides gives benzylthiophene and fluoreno[3,4- $b]$ thiophene products

\section{Graphical Abstract}<smiles>COc1ccsc1C(=O)C(=Pc1ccccc1)c1ccccc1</smiles><smiles>CCC(=Pc1ccccc1)C(=O)c1cscc1OC</smiles><smiles>C=Cc1cc2cscc2o1</smiles><smiles>CS(=O)(=O)c1ccsc1-c1ccccc1</smiles>

$45 \%$

$25 \%$<smiles></smiles><smiles>[2H]C([2H])=Cc1c([2H])csc1-c1ccccc1</smiles><smiles>Cc1ccsc1/C=C/C(=O)C(=P)c1ccccc1OC(C)(F)F</smiles>
FVP, $800^{\circ} \mathrm{C}$<smiles>O=C(c1ccccc1)c1ccc2sccc2c1</smiles><smiles>O=C(c1ccccc1)C(c1ccccc1)C(c1ccccc1)c1ccccc1C1=Cc2c1ccc1sccc21</smiles> 